\title{
Integrated 6-DOF Orbit-Attitude Dynamical Modeling and Control Using Geometric Mechanics
}

\author{
Ling Jiang, Yue Wang, and Shijie Xu \\ School of Astronautics, Beihang University, Beijing 100191, China \\ Correspondence should be addressed to Yue Wang; ywang@buaa.edu.cn
}

Received 6 March 2017; Accepted 8 May 2017; Published 30 May 2017

Academic Editor: Enrico C. Lorenzini

Copyright (C) 2017 Ling Jiang et al. This is an open access article distributed under the Creative Commons Attribution License, which permits unrestricted use, distribution, and reproduction in any medium, provided the original work is properly cited.

\begin{abstract}
The integrated 6-DOF orbit-attitude dynamical modeling and control have shown great importance in various missions, for example, formation flying and proximity operations. The integrated approach yields better performances than the separate one in terms of accuracy, efficiency, and agility. One challenge in the integrated approach is to find a unified representation for the 6-DOF motion with configuration space SE(3). Recently, exponential coordinates of SE(3) have been used in dynamics and control of the 6-DOF motion, however, only on the kinematical level. In this paper, we will improve the current method by adopting exponential coordinates on the dynamical level, by giving the relation between the second-order derivative of exponential coordinates and spacecraft's accelerations. In this way, the 6-DOF motion in terms of exponential coordinates can be written as a second-order system with a quite compact form, to which a broader range of control theories, such as higher-order sliding modes, can be applied. For a demonstration purpose, a simple asymptotic tracking control law with almost global convergence is designed. Finally, the integrated modeling and control are applied to the body-fixed hovering over an asteroid and verified by a simulation, in which absolute motions of the spacecraft and asteroid are simulated separately.
\end{abstract}

\section{Introduction}

The integrated 6-DOF orbit-attitude dynamical modeling and control of spacecraft have shown great importance in various space missions, such as formation flying [1-7], proximity operations [8-14], and proximity operations about minor celestial bodies [15-17].

Necessity of the integrated approach for spacecraft's 6DOF motion lies in two aspects. Firstly, the orbit and attitude motions are actually kinematically coupled in spacecraft relative motions $[1,7]$ and are dynamically coupled due to effects of external forces and torques, such as the gravitational orbitattitude coupling in close proximity of minor celestial bodies [18-21] and the orbit-attitude coupling of high area-to-mass ratio (HAMR) objects caused by the solar radiation pressure (SRP) $[22,23]$. Secondly, and more importantly, proximity operations and some formation flying missions, such as proximity rendezvous, docking, landing on and sampling an asteroid, interferometric observation, and coordinated pointing, usually require the relative position and attitude of spacecraft to follow the desired trajectory simultaneously.
Unlike the traditional approach that treats orbit and attitude motions separately, such as Zhang et al. [24, 25], the integrated approach models and controls the orbit and attitude motions in a unified framework. Therefore, integrated orbit-attitude dynamical modeling and control will capture the system's dynamics better and will lead the 6-DOF motion in the phase space more accurately and effectively. That is, the integrated approach yields better performances than the separate one in terms of accuracy, efficiency, and agility.

There are two main challenges in the integrated dynamical modeling and control. The first one is to find an adequate unified mathematical representation for the 6-DOF orbitattitude motion, which must be amenable for the application of various nonlinear control theories. The unified representation of the 6-DOF motion is also required to be applicable to a wide range of spacecraft maneuvers.

The second challenge is that the 6-DOF orbit-attitude motion is highly nonlinear and is subjected to external disturbances and parameter uncertainties. Consequently, adequate control theories are needed in the controller design, such as the sliding mode control [9], adaptive control [12, 13], 
adaptive sliding mode control $[3,17]$, adaptive terminal sliding mode control $[2,14]$, finite-time control $[5,16,26]$, and state-dependent Riccati equation (SDRE) method $[4,7]$.

As for the unified mathematical representation of the 6DOF motion, the spacecraft can be considered as a rigid body and the configuration space is the Lie group SE(3), the special Euclidean group, which is the set of position and attitude of a rigid body moving in a three-dimensional Euclidean space. The dual quaternion has been widely used as a unified representation of the 6-DOF rigid body motion, but the constraints between its elements need to be dealt with carefully $[10,11]$. Sanyal et al. $[8,27]$ have used the matrix form of the Lie group SE(3) in the dynamical modeling of rigid body motion and achieved controllers with almost global convergence, which is the best that can be achieved for a system evolving on a noncontractible state space, like the 6-DOF rigid body motion, with continuous feedback [6]. However, due to the complexity of matrix calculations, the matrix form of $\mathrm{SE}(3)$ is not convenient for controller design.

Recently, exponential coordinates of Lie group SE(3) have been used as a unified representation of the 6-DOF orbit-attitude (rigid body) motion in spacecraft formation flying and asteroid hovering [6, 9, 14-17]. The exponential coordinates are in vector form with six elements, and thus, unlike the dual quaternion, have no constraint between elements. Besides, compared with the matrix form of SE(3), the set of exponential coordinates with a vector form is more convenient for applications of nonlinear control theories. In the mentioned studies, the exponential coordinates are used to describe the 6-DOF kinematics of the spacecraft, and the control scheme is designed to reduce errors of configuration and velocities from almost any given initial state, except those that differ in orientation by a $\pi$ radian rotation from the desired states where exponential coordinates for the attitude are not uniquely defined [6]. Because the set of such initial states is an embedded lower-dimensional subspace of the state space, the tracking control scheme designed in terms of exponential coordinates can achieve almost global convergence, which makes it applicable in practice. Exponential coordinates of SE(3) used by Lee et al. $[6,15,16]$ have provided a good unified representation for the 6-DOF motion with several advantages: having a vector form, having no constraint between elements, being convenient for controller design, and achieving almost global convergence in the controller.

In the present paper, based on the results by Bullo and Murray [28], we will improve the dynamical modeling method for the 6-DOF rigid body motion suggested by Lee et al. $[6,15,16]$. Lee et al. used exponential coordinates of $\operatorname{SE}(3)$ only in the kinematics of spacecraft, that is, only gave the relation between the first-order time derivative of exponential coordinates and the (angular) velocity of spacecraft. We will use exponential coordinates of SE(3) on the dynamical level further, that is, give the relation between the second-order time derivative of exponential coordinates and the (angular) acceleration of spacecraft. By using this relation on the dynamical level, the system can be written as a second-order system with a compact form. Then, the set of exponential coordinates becomes more convenient for controller design

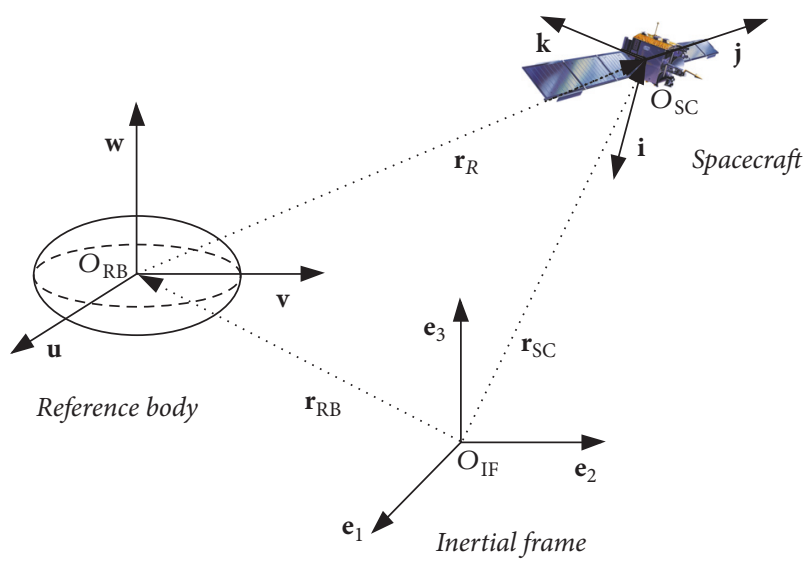

FIGURE 1: The spacecraft moving in proximity of the reference body.

and a broader range of control theories, such as higher-order sliding modes, can be applied.

Another improvement suggested in the current work is to introduce a desired trajectory for the 6-DOF motion, that is, the guidance on $\mathrm{SE}(3)$. The controller will lead the system to follow the desired trajectory until reaching the desired final state. In the earlier studies by Lee et al. [6, 15, 16], Lee [9], and Lee and Vukovich $[14,17]$, the controller aimed at the desired final state directly. Therefore, the true trajectory to the final state could not be specified. Compared with Lee's approach, our approach has two advantages: firstly, it is applicable to diverse spacecraft proximity operations requiring a specific 6-DOF orbit-attitude trajectory, for example, approaching along an obstacle-free trajectory; secondly, the initial tracking error is much smaller and the control saturation in the beginning can be avoided.

Based on the second-order system in terms of exponential coordinates of SE(3), a simple asymptotic tracking control law with almost global convergence is designed for a demonstration purpose. Finally, both the integrated dynamical modeling and control law are applied to body-fixed orbitattitude hovering over an asteroid.

\section{Integrated Modeling of 6-DOF Relative Dynamics}

As shown in Figure 1, the 6-DOF orbit-attitude (rigid body) motion of a spacecraft in proximity of the reference body is considered. The reference body can be another spacecraft in spacecraft formation flying and proximity operations or the asteroid in minor celestial body proximity operations. The inertial frame is denoted by $S_{\mathrm{IF}}=\left\{\mathbf{e}_{1}, \mathbf{e}_{2}, \mathbf{e}_{3}\right\}$ with $O_{\mathrm{IF}}$ as its origin. The body-fixed principal-axis frames of the reference body and spacecraft are denoted by $S_{\mathrm{RB}}=\{\mathbf{u}, \mathbf{v}, \mathbf{w}\}$ and $S_{\mathrm{SC}}=$ $\{\mathbf{i}, \mathbf{j}, \mathbf{k}\}$ with $O_{\mathrm{RB}}$ and $O_{\mathrm{SC}}$ as their origins, respectively.

2.1. Dynamics of Spacecraft. The attitude of the spacecraft with respect to the inertial frame $S_{\mathrm{IF}}$ is described by matrix $\mathbf{A}_{\mathrm{SC}}$ :

$$
\mathbf{A}_{\mathrm{SC}}=\left[\boldsymbol{\alpha}_{\mathrm{SC}}, \boldsymbol{\beta}_{\mathrm{SC}}, \boldsymbol{\gamma}_{\mathrm{SC}}\right]^{T} \in \mathrm{SO}(3),
$$


where $\boldsymbol{\alpha}_{\mathrm{SC}}, \boldsymbol{\beta}_{\mathrm{SC}}$, and $\boldsymbol{\gamma}_{\mathrm{SC}}$ are coordinates of unit vectors $\mathbf{e}_{1}$, $\mathbf{e}_{2}$, and $\mathbf{e}_{3}$ of the inertial frame $S_{\mathrm{IF}}$ expressed in the bodyfixed frame of spacecraft $S_{\mathrm{SC}}$, respectively. Matrix $\mathbf{A}_{\mathrm{SC}}$ is also the coordinate transformation matrix from the bodyfixed frame $S_{\mathrm{SC}}$ to the inertial frame $S_{\mathrm{IF}}$. SO(3) is the threedimensional special orthogonal group. The position vector of the spacecraft with respect to the origin $O_{\mathrm{IF}}$ expressed in the inertial frame $S_{\mathrm{IF}}$ is denoted by $\mathbf{r}_{\mathrm{SC}}$.

The configuration space of 6-DOF orbit-attitude motion of the spacecraft is the Lie group:

$$
Q_{\mathrm{SC}}=\mathrm{SE}(3) \text {, }
$$

known as the special Euclidean group of three-dimensional space with elements $\left(\mathbf{A}_{\mathrm{SC}}, \mathbf{r}_{\mathrm{SC}}\right)$, which is the semidirect product of $\mathrm{SO}(3)$ and $\mathbb{R}^{3}$, that is, $\mathrm{SO}(3) \ltimes \mathbb{R}^{3}$. The velocity phase space of 6-DOF motion of the spacecraft is the tangent bundle TSE(3), which can be represented by $\operatorname{SE}(3) \times \operatorname{se}(3)$ through the left translation, where se(3) is the Lie algebra of Lie group $\mathrm{SE}(3)$.

The configuration of spacecraft on the special Euclidean group $\mathrm{SE}(3)$ can be represented by the following $4 \times 4$ matrix:

$$
\mathbf{g}_{\mathrm{SC}}=\left[\begin{array}{cc}
\mathbf{A}_{\mathrm{SC}} & \mathbf{r}_{\mathrm{SC}} \\
\mathbf{0} & 1
\end{array}\right] \in \mathrm{SE}(3) \text {. }
$$

The velocities of spacecraft, which are contained within Lie algebra se(3), can be represented by the $6 \times 1$ vector:

$$
\boldsymbol{\xi}_{\mathrm{SC}}=\left[\begin{array}{l}
\boldsymbol{\Omega}_{\mathrm{SC}} \\
\mathbf{V}_{\mathrm{SC}}
\end{array}\right] \in \mathbb{R}^{6},
$$

where $\boldsymbol{\Omega}_{\mathrm{SC}}$ and $\mathbf{V}_{\mathrm{SC}}$ are the spacecraft's angular velocity and velocity with respect to the inertial frame, respectively, both expressed in its body-fixed frame $S_{\mathrm{SC}}$.

Then, the 6-DOF orbit-attitude kinematics of the spacecraft can be given by [15]

$$
\dot{\mathbf{g}}_{\mathrm{SC}}=\mathbf{g}_{\mathrm{SC}}\left(\boldsymbol{\xi}_{\mathrm{SC}}\right)^{\vee} \text {, }
$$

where the map $(\cdot)^{\vee}: \mathbb{R}^{6} \rightarrow \operatorname{se}(3)$ is the Lie algebra isomorphism defined by

$$
\left(\boldsymbol{\xi}_{\mathrm{SC}}\right)^{\vee}=\left[\begin{array}{cc}
\left(\boldsymbol{\Omega}_{\mathrm{SC}}\right)^{\times} & \mathbf{V}_{\mathrm{SC}} \\
\mathbf{0} & 0
\end{array}\right] \in \operatorname{se}(3) .
$$

The map $(\cdot)^{\times}: \mathbb{R}^{3} \rightarrow \operatorname{so}(3)$ is the Lie algebra isomorphism, which is also the cross-product operator for $3 \times 1$ vectors, defined by

$$
(\mathbf{w})^{\times}=\left[\begin{array}{l}
w^{x} \\
w^{y} \\
w^{z}
\end{array}\right]^{\times}=\left[\begin{array}{ccc}
0 & -w^{z} & w^{y} \\
w^{z} & 0 & -w^{x} \\
-w^{y} & w^{x} & 0
\end{array}\right] \in \operatorname{so}(3),
$$

where so(3) is the Lie algebra of Lie group $\mathrm{SO}(3)$.

The 6-DOF orbit-attitude dynamics of the spacecraft can be given by [15]

$$
\rrbracket_{\mathrm{SC}} \dot{\boldsymbol{\xi}}_{\mathrm{SC}}=\operatorname{ad}_{\xi_{\mathrm{SC}}}^{*}\left(\rrbracket_{\mathrm{SC}} \boldsymbol{\xi}_{\mathrm{SC}}\right)+\boldsymbol{\tau}_{\mathrm{SC}}^{n}+\boldsymbol{\tau}_{\mathrm{SC}}^{c}
$$

where $\rrbracket_{S C}$ is the combined matrix of the mass and inertia of the spacecraft given by

$$
\rrbracket_{\mathrm{SC}}=\left[\begin{array}{cc}
\mathbf{I}_{\mathrm{SC}} & \mathbf{0} \\
\mathbf{0} & m_{\mathrm{SC}} \mathbf{I}_{3 \times 3}
\end{array}\right] \in \mathbb{R}^{6 \times 6},
$$

$\mathbf{I}_{\mathrm{SC}}$ and $m_{\mathrm{SC}}$ are the inertia tensor and mass of the spacecraft, respectively, and $\mathbf{I}_{3 \times 3}$ is the $3 \times 3$ identity matrix. The adjoint operator $\mathrm{ad}_{\xi_{\mathrm{SC}}}$ and coadjoint operator $\mathrm{ad}_{\xi_{\mathrm{SC}}}^{*}$ on the Lie algebra se(3) can be given as matrixes:

$$
\begin{aligned}
\operatorname{ad}_{\boldsymbol{\xi}_{\mathrm{SC}}} & =\left[\begin{array}{cc}
\left(\boldsymbol{\Omega}_{\mathrm{SC}}\right)^{\times} & \mathbf{0} \\
\left(\mathbf{V}_{\mathrm{SC}}\right)^{\times} & \left(\boldsymbol{\Omega}_{\mathrm{SC}}\right)^{\times}
\end{array}\right] \in \mathbb{R}^{6 \times 6}, \\
\operatorname{ad}_{\boldsymbol{\xi}_{\mathrm{SC}}}^{*} & =\left(\operatorname{ad}_{\xi_{\mathrm{SC}}}\right)^{T}=\left[\begin{array}{cc}
-\left(\boldsymbol{\Omega}_{\mathrm{SC}}\right)^{\times} & -\left(\mathbf{V}_{\mathrm{SC}}\right)^{\times} \\
\mathbf{0} & -\left(\boldsymbol{\Omega}_{\mathrm{SC}}\right)^{\times}
\end{array}\right] \in \mathbb{R}^{6 \times 6} .
\end{aligned}
$$

The environmental torque $\mathbf{T}_{\mathrm{SC}}^{n}$ and force $\mathbf{F}_{\mathrm{SC}}^{n}$ acting on the spacecraft, both expressed in the body-fixed frame $S_{\mathrm{SC}}$, are written together in vector $\tau_{\mathrm{SC}}^{n}$ :

$$
\boldsymbol{\tau}_{\mathrm{SC}}^{n}=\left[\begin{array}{c}
\mathbf{T}_{\mathrm{SC}}^{n} \\
\mathbf{F}_{\mathrm{SC}}^{n}
\end{array}\right] \in \mathbb{R}^{6} .
$$

Keep in mind that, in minor celestial body proximity operations, the environmental torque and force $\boldsymbol{\tau}_{\mathrm{SC}}^{n}$ include the gravitational torque and force by the minor body, while, in spacecraft formation flying and proximity operations, the gravitational torque and force between two spacecraft will be neglected.

The control torque $\mathbf{T}_{\mathrm{SC}}^{c}$ and force $\mathbf{F}_{\mathrm{SC}}^{c}$ acting on the spacecraft, both expressed in the body-fixed frame $S_{\mathrm{SC}}$, are written together in vector $\tau_{\mathrm{SC}}^{c}$ :

$$
\boldsymbol{\tau}_{\mathrm{SC}}^{c}=\left[\begin{array}{l}
\mathbf{T}_{\mathrm{SC}}^{c} \\
\mathbf{F}_{\mathrm{SC}}^{c}
\end{array}\right] \in \mathbb{R}^{6} .
$$

2.2. Dynamics of Reference Body. The 6-DOF orbit-attitude dynamics of the reference body is described similarly as that of the spacecraft. The attitude of reference body with respect to the inertial frame $S_{\mathrm{IF}}$ is described by matrix $\mathrm{A}_{\mathrm{RB}}$ :

$$
\mathbf{A}_{\mathrm{RB}}=\left[\boldsymbol{\alpha}_{\mathrm{RB}}, \boldsymbol{\beta}_{\mathrm{RB}}, \boldsymbol{\gamma}_{\mathrm{RB}}\right]^{T} \in \mathrm{SO}(3),
$$

where $\boldsymbol{\alpha}_{\mathrm{RB}}, \boldsymbol{\beta}_{\mathrm{RB}}$, and $\boldsymbol{\gamma}_{\mathrm{RB}}$ are coordinates of unit vectors $\mathbf{e}_{1}, \mathbf{e}_{2}$, and $\mathbf{e}_{3}$ expressed in the body-fixed frame of reference body $S_{\mathrm{RB}}$, respectively. The position vector of the reference body with respect to the origin $O_{\mathrm{IF}}$ expressed in the inertial frame $S_{\mathrm{IF}}$ is denoted by $\mathbf{r}_{\mathrm{RB}}$. The configuration space of reference body, $Q_{R B}$, is also Lie group $S E(3)$ with elements $\left(\mathbf{A}_{\mathrm{RB}}, \mathbf{r}_{\mathrm{RB}}\right)$. The configuration of the reference body is denoted by

$$
\mathbf{g}_{\mathrm{RB}}=\left[\begin{array}{cc}
\mathbf{A}_{\mathrm{RB}} & \mathbf{r}_{\mathrm{RB}} \\
\mathbf{0} & 1
\end{array}\right] \in \mathrm{SE}(3) .
$$

The velocities of the reference body are represented by

$$
\boldsymbol{\xi}_{\mathrm{RB}}=\left[\begin{array}{l}
\boldsymbol{\Omega}_{\mathrm{RB}} \\
\mathbf{V}_{\mathrm{RB}}
\end{array}\right] \in \mathbb{R}^{6},
$$


where $\boldsymbol{\Omega}_{\mathrm{RB}}$ and $\mathbf{V}_{\mathrm{RB}}$ are angular velocity and velocity of the reference body with respect to the inertial frame, respectively, both expressed in the reference body's body-fixed frame $S_{\mathrm{RB}}$.

The 6-DOF orbit-attitude kinematics of the reference body is given by

$$
\dot{\mathbf{g}}_{\mathrm{RB}}=\mathbf{g}_{\mathrm{RB}}\left(\boldsymbol{\xi}_{\mathrm{RB}}\right)^{\vee},
$$

where

$$
\left(\boldsymbol{\xi}_{\mathrm{RB}}\right)^{\vee}=\left[\begin{array}{cc}
\left(\boldsymbol{\Omega}_{\mathrm{RB}}\right)^{\times} & \mathbf{V}_{\mathrm{RB}} \\
\mathbf{0} & 0
\end{array}\right] \in \operatorname{se}(3) .
$$

The 6-DOF orbit-attitude dynamics of the reference body can be given by

$$
\rrbracket_{\mathrm{RB}} \dot{\xi}_{\mathrm{RB}}=\operatorname{ad}_{\xi_{\mathrm{RB}}}^{*}\left(\rrbracket_{\mathrm{RB}} \xi_{\mathrm{RB}}\right)+\tau_{\mathrm{RB}},
$$

where $\square_{\mathrm{RB}}$ is the combined matrix of the mass and inertia of the reference body

$$
\mathbb{R}_{\mathrm{RB}}=\left[\begin{array}{cc}
\mathbf{I}_{\mathrm{RB}} & \mathbf{0} \\
\mathbf{0} & m_{\mathrm{RB}} \mathbf{I}_{3 \times 3}
\end{array}\right] \in \mathbb{R}^{6 \times 6},
$$

$\mathbf{I}_{\mathrm{RB}}$ and $m_{\mathrm{RB}}$ are the inertia tensor and mass of the reference body, respectively, and

$$
\operatorname{ad}_{\boldsymbol{\xi}_{\mathrm{RB}}}^{*}=\left[\begin{array}{cc}
-\left(\boldsymbol{\Omega}_{\mathrm{RB}}\right)^{\times} & -\left(\mathbf{V}_{\mathrm{RB}}\right)^{\times} \\
\mathbf{0} & -\left(\boldsymbol{\Omega}_{\mathrm{RB}}\right)^{\times}
\end{array}\right] \in \mathbb{R}^{6 \times 6} .
$$

Resultant torque $\mathbf{T}_{\mathrm{RB}}$ and force $\mathbf{F}_{\mathrm{RB}}$ acting on the reference body, both expressed in the body-fixed frame $S_{\mathrm{RB}}$, are written together in vector $\tau_{\mathrm{RB}}$ :

$$
\boldsymbol{\tau}_{\mathrm{RB}}=\left[\begin{array}{c}
\mathbf{T}_{\mathrm{RB}} \\
\mathbf{F}_{\mathrm{RB}}
\end{array}\right] \in \mathbb{R}^{6} .
$$

The torque and force $\boldsymbol{\tau}_{\mathrm{RB}}$ will include control torque and force acting on the reference body, if they exist, in spacecraft formation flying and proximity operations.

2.3. Relative Dynamics of Spacecraft. In space missions, what really matters is the relative dynamics of spacecraft with respect to the reference body, rather than the absolute dynamics of spacecraft in the inertial space. The 6-DOF relative dynamics of spacecraft, which will be written in a similar form with the absolute dynamics, can be obtained based on the results in Sections 2.1 and 2.2.

The relative configuration of spacecraft with respect to the reference body, which is also on the special Euclidean group $\mathrm{SE}(3)$, is denoted by

$$
\mathbf{g}_{R}=\left(\mathbf{g}_{\mathrm{RB}}\right)^{-1} \mathbf{g}_{\mathrm{SC}}=\left[\begin{array}{cc}
\mathbf{A}_{R} & \mathbf{r}_{R} \\
\mathbf{0} & 1
\end{array}\right] \in \mathrm{SE}(3),
$$

where matrix $\mathbf{A}_{R}$ is the relative attitude of spacecraft with respect to the reference body, which is also the coordinate transformation matrix from the body-fixed frame of spacecraft $S_{\mathrm{SC}}$ to the body-fixed frame of reference body $S_{\mathrm{RB}}$, and vector $\mathbf{r}_{R}$ is the relative position of spacecraft with respect to the reference body $\mathrm{O}_{\mathrm{RB}}$, expressed in the body-fixed frame of reference body $S_{\mathrm{RB}}$.

The relative attitude matrix $\mathbf{A}_{R}$ can be written as

$$
\mathbf{A}_{R}=\left[\boldsymbol{\alpha}_{R}, \boldsymbol{\beta}_{R}, \boldsymbol{\gamma}_{R}\right]^{T},
$$

where $\boldsymbol{\alpha}_{R}, \boldsymbol{\beta}_{R}$, and $\boldsymbol{\gamma}_{R}$ are coordinates of unit vectors $\mathbf{u}, \mathbf{v}$, and $\mathbf{w}$ of the reference body's body-fixed frame $S_{\mathrm{RB}}$ expressed in the spacecraft's body-fixed frame $S_{\mathrm{SC}}$, respectively.

According to (3) and (14), the relative configuration of spacecraft can be obtained as

$$
\begin{aligned}
\mathbf{g}_{R} & =\left(\mathbf{g}_{\mathrm{RB}}\right)^{-1} \mathbf{g}_{\mathrm{SC}}=\left[\begin{array}{cc}
\mathbf{A}_{\mathrm{RB}}^{-1} & -\mathbf{A}_{\mathrm{RB}}^{-1} \mathbf{r}_{\mathrm{RB}} \\
\mathbf{0} & 1
\end{array}\right]\left[\begin{array}{cc}
\mathbf{A}_{\mathrm{SC}} & \mathbf{r}_{\mathrm{SC}} \\
\mathbf{0} & 1
\end{array}\right] \\
& =\left[\begin{array}{cc}
\mathbf{A}_{\mathrm{RB}}^{-1} \mathbf{A}_{\mathrm{SC}} & \mathbf{A}_{\mathrm{RB}}^{-1}\left(\mathbf{r}_{\mathrm{SC}}-\mathbf{r}_{\mathrm{RB}}\right) \\
\mathbf{0} & 1
\end{array}\right] .
\end{aligned}
$$

Then, the 6-DOF relative orbit-attitude kinematics of the spacecraft with respect to the reference body can be given by

$$
\begin{aligned}
\dot{\mathbf{g}}_{R}= & \left(\mathbf{g}_{\mathrm{RB}}\right)^{-1} \dot{\mathbf{g}}_{\mathrm{SC}}+\frac{d}{d t}\left[\left(\mathbf{g}_{\mathrm{RB}}\right)^{-1}\right] \mathbf{g}_{\mathrm{SC}} \\
= & \left(\mathbf{g}_{\mathrm{RB}}\right)^{-1} \dot{\mathbf{g}}_{\mathrm{SC}}-\left(\mathbf{g}_{\mathrm{RB}}\right)^{-1} \dot{\mathbf{g}}_{\mathrm{RB}}\left(\mathbf{g}_{\mathrm{RB}}\right)^{-1} \mathbf{g}_{\mathrm{SC}} \\
= & \left(\mathbf{g}_{\mathrm{RB}}\right)^{-1} \mathbf{g}_{\mathrm{SC}}\left(\boldsymbol{\xi}_{\mathrm{SC}}\right)^{\vee} \\
& -\left(\mathbf{g}_{\mathrm{RB}}\right)^{-1} \mathbf{g}_{\mathrm{RB}}\left(\boldsymbol{\xi}_{\mathrm{RB}}\right)^{\vee}\left(\mathbf{g}_{\mathrm{RB}}\right)^{-1} \mathbf{g}_{\mathrm{SC}} \\
= & \mathbf{g}_{R}\left(\boldsymbol{\xi}_{\mathrm{SC}}\right)^{\vee}-\left(\boldsymbol{\xi}_{\mathrm{RB}}\right)^{\vee} \mathbf{g}_{R} \\
= & \mathbf{g}_{R}\left[\left(\xi_{\mathrm{SC}}\right)^{\vee}-\left(\mathbf{g}_{R}\right)^{-1}\left(\boldsymbol{\xi}_{\mathrm{RB}}\right)^{\vee} \mathbf{g}_{R}\right] .
\end{aligned}
$$

The relative kinematics of spacecraft (25) can be written in the same form as the absolute kinematics (5) and (16):

$$
\dot{\mathbf{g}}_{R}=\mathbf{g}_{R}\left(\boldsymbol{\xi}_{R}\right)^{\vee}
$$

where the $6 \times 1$ vector $\xi_{R}$ is the relative velocity of spacecraft:

$$
\boldsymbol{\xi}_{R}=\left[\begin{array}{l}
\boldsymbol{\Omega}_{R} \\
\mathbf{V}_{R}
\end{array}\right] \in \mathbb{R}^{6} .
$$

Vectors $\boldsymbol{\Omega}_{R}$ and $\mathbf{V}_{R}$ are relative angular velocity and relative velocity of spacecraft with respect to the reference body, respectively, both expressed in the body-fixed frame of spacecraft $S_{\mathrm{SC}}$.

According to (25) and (26), we can obtain the relative velocity of spacecraft $\left(\xi_{R}\right)^{\vee}$ as follows:

$$
\left(\boldsymbol{\xi}_{R}\right)^{\vee}=\left(\boldsymbol{\xi}_{\mathrm{SC}}\right)^{\vee}-\left(\mathbf{g}_{R}\right)^{-1}\left(\boldsymbol{\xi}_{\mathrm{RB}}\right)^{\vee} \mathbf{g}_{R}
$$

which can be written in a compact form

$$
\boldsymbol{\xi}_{R}=\boldsymbol{\xi}_{\mathrm{SC}}-\mathrm{Ad}_{\left(\mathrm{g}_{R}\right)^{-1}} \boldsymbol{\xi}_{\mathrm{RB}} .
$$

The operator $\operatorname{Ad}_{\left(\mathbf{g}_{R}\right)^{-1}}$, the adjoint action of $\left(\mathbf{g}_{R}\right)^{-1} \in \mathrm{SE}(3)$ on $\left(\xi_{\mathrm{RB}}\right)^{\vee} \in \operatorname{se}(3)$, satisfies

$$
\left(\operatorname{Ad}_{\left(\mathbf{g}_{R}\right)^{-1}} \boldsymbol{\xi}_{\mathrm{RB}}\right)^{\vee}=\left(\mathbf{g}_{R}\right)^{-1}\left(\boldsymbol{\xi}_{\mathrm{RB}}\right)^{\vee} \mathbf{g}_{R}
$$


and the matrix form of operator $\operatorname{Ad}_{\left(\mathbf{g}_{R}\right)^{-1}}$ can be given by

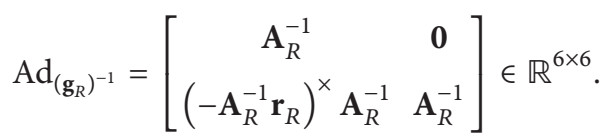

As for the 6-DOF relative orbit-attitude dynamics of the spacecraft with respect to the reference body, the time derivative of relative velocity $\boldsymbol{\xi}_{R}$, that is, the relative acceleration of the spacecraft, can be obtained according to (29):

$$
\dot{\boldsymbol{\xi}}_{R}=\dot{\boldsymbol{\xi}}_{\mathrm{SC}}-\frac{d}{d t}\left[\operatorname{Ad}_{\left(\mathrm{g}_{R}\right)^{-1}} \boldsymbol{\xi}_{\mathrm{RB}}\right]
$$

According to Lee et al. [15],

$$
\frac{d}{d t}\left[\operatorname{Ad}_{\left(\mathbf{g}_{R}\right)^{-1}} \boldsymbol{\xi}_{\mathrm{RB}}\right]=-\operatorname{ad}_{\xi_{R}} \operatorname{Ad}_{\left(\mathbf{g}_{R}\right)^{-1}} \boldsymbol{\xi}_{\mathrm{RB}}+\operatorname{Ad}_{\left(\mathbf{g}_{R}\right)^{-1}} \dot{\boldsymbol{\xi}}_{\mathrm{RB}},
$$

where the adjoint operator $\operatorname{ad}_{\xi_{R}}$ on the Lie algebra se(3) is defined in (10).

Therefore, the relative acceleration of the spacecraft $\dot{\xi}_{R}$ can be written as

$$
\dot{\boldsymbol{\xi}}_{R}=\dot{\boldsymbol{\xi}}_{\mathrm{SC}}+\operatorname{ad}_{\xi_{R}} \operatorname{Ad}_{\left(\mathrm{g}_{R}\right)^{-1}} \boldsymbol{\xi}_{\mathrm{RB}}-\operatorname{Ad}_{\left(\mathrm{g}_{R}\right)^{-1}} \dot{\xi}_{\mathrm{RB}}
$$

According to (8) and (34), the 6-DOF relative dynamics of the spacecraft with respect to the reference body can be given by

$$
\begin{aligned}
\rrbracket_{\mathrm{SC}} \dot{\boldsymbol{\xi}}_{R}= & \rrbracket_{\mathrm{SC}} \dot{\boldsymbol{\xi}}_{\mathrm{SC}}+\rrbracket_{\mathrm{SC}}\left[\operatorname{ad}_{\xi_{R}} \operatorname{Ad}_{\left(\mathrm{g}_{R}\right)^{-1}} \boldsymbol{\xi}_{\mathrm{RB}}-\operatorname{Ad}_{\left(\mathrm{g}_{R}\right)^{-1}} \dot{\boldsymbol{\xi}}_{\mathrm{RB}}\right] \\
= & \operatorname{ad}_{\xi_{\mathrm{SC}}}^{*} \rrbracket_{\mathrm{SC}} \boldsymbol{\xi}_{\mathrm{SC}}+\boldsymbol{\tau}_{\mathrm{SC}}^{n}+\boldsymbol{\tau}_{\mathrm{SC}}^{c} \\
& +\rrbracket_{\mathrm{SC}}\left\{\operatorname{ad}_{\xi_{R}} \operatorname{Ad}_{\left(\mathrm{g}_{R}\right)^{-1}} \boldsymbol{\xi}_{\mathrm{RB}}-\mathrm{Ad}_{\left(\mathrm{g}_{R}\right)^{-1}} \dot{\boldsymbol{\xi}}_{\mathrm{RB}}\right\} .
\end{aligned}
$$

Equations (26) and (35) form complete equations for the 6DOF relative motion.

\section{Tracking Error Modeling and Control Law Design}

In formation flying and proximity operations, the 6-DOF relative orbit-attitude motion of the spacecraft with respect to the reference body is usually required to track the desired trajectory. The goal of the controller is to reduce the tracking error. Here, introducing a desired trajectory for the 6-DOF orbit-attitude motion, that is, guidance on $\mathrm{SE}(3)$, is an improvement compared with the earlier works by Lee et al. $[6,15,16]$ and Lee and Vukovich $[14,17]$, where the controllers aimed at the desired final state directly. In our approach with a reference trajectory, the true orbit-attitude trajectory to the final state can be specified, and the control saturation caused by the large initial tracking error can be avoided.

3.1. Desired Relative Trajectory and Tracking Error. The desired trajectory is denoted by $\left(\mathbf{g}_{d}(t), \boldsymbol{\xi}_{d}(t), \dot{\boldsymbol{\xi}}_{d}(t)\right)$, where

$$
\dot{\mathbf{g}}_{d}=\mathbf{g}_{d}\left(\boldsymbol{\xi}_{d}\right)^{\vee} .
$$

The goal of the orbit-attitude motion controller is $\left(\mathbf{g}_{R}(t)\right.$, $\left.\boldsymbol{\xi}_{R}(t)\right) \rightarrow\left(\mathbf{g}_{d}(t), \boldsymbol{\xi}_{d}(t)\right)$.
The tracking error in configuration space of the relative motion, which is also on the special Euclidean group SE(3), is defined by

$$
\mathbf{g}_{e}=\left(\mathbf{g}_{d}\right)^{-1} \mathbf{g}_{R}=\left[\begin{array}{cc}
\mathbf{A}_{e} & \mathbf{r}_{e} \\
\mathbf{0} & 1
\end{array}\right] \in \mathrm{SE}(3) .
$$

By using the same method as in Section 2.3, the kinematics of tracking error of the spacecraft's relative motion is given by

$$
\dot{\mathbf{g}}_{e}=\mathbf{g}_{e}\left(\xi_{e}\right)^{\vee},
$$

where the $6 \times 1$ vector $\xi_{e}$ is the tracking error in the relative velocity:

$$
\boldsymbol{\xi}_{e}=\left[\begin{array}{l}
\boldsymbol{\Omega}_{e} \\
\mathbf{V}_{e}
\end{array}\right]=\boldsymbol{\xi}_{R}-\operatorname{Ad}_{\left(\mathbf{g}_{e}\right)^{-1}} \boldsymbol{\xi}_{d} .
$$

The time derivative of velocity tracking error $\xi_{e}$ can be obtained:

$$
\dot{\boldsymbol{\xi}}_{e}=\dot{\boldsymbol{\xi}}_{R}+\operatorname{ad}_{\xi_{e}} \operatorname{Ad}_{\left(\mathbf{g}_{e}\right)^{-1}} \boldsymbol{\xi}_{d}-\operatorname{Ad}_{\left(\mathbf{g}_{e}\right)^{-1}} \dot{\boldsymbol{\xi}}_{d} .
$$

According to (35), the dynamics of the 6-DOF orbitattitude tracking error can be given by

$$
\begin{gathered}
\rrbracket_{\mathrm{SC}} \dot{\boldsymbol{\xi}}_{e}=\operatorname{ad}_{\xi_{\mathrm{SC}}}^{*} \rrbracket_{\mathrm{SC}} \boldsymbol{\xi}_{\mathrm{SC}}+\boldsymbol{\tau}_{\mathrm{SC}}^{n}+\boldsymbol{\tau}_{\mathrm{SC}}^{c}+\rrbracket_{\mathrm{SC}}\left\{\operatorname{ad}_{\boldsymbol{\xi}_{e}} \operatorname{Ad}_{\left(\mathrm{g}_{e}\right)^{-1}} \boldsymbol{\xi}_{d}\right. \\
\left.-\operatorname{Ad}_{\left(\mathbf{g}_{e}\right)^{-1}} \dot{\boldsymbol{\xi}}_{d}+\operatorname{ad}_{\boldsymbol{\xi}_{R}} \operatorname{Ad}_{\left(\mathrm{g}_{R}\right)^{-1}} \boldsymbol{\xi}_{\mathrm{RB}}-\operatorname{Ad}_{\left(\mathrm{g}_{R}\right)^{-1}} \dot{\boldsymbol{\xi}}_{\mathrm{RB}}\right\}
\end{gathered}
$$

where the motion of reference body $\left(\mathbf{g}_{\mathrm{RB}}(t), \boldsymbol{\xi}_{\mathrm{RB}}(t), \dot{\boldsymbol{\xi}}_{\mathrm{RB}}(t)\right)$ and the desired relative motion $\left(\mathbf{g}_{d}(t), \boldsymbol{\xi}_{d}(t), \dot{\boldsymbol{\xi}}_{d}(t)\right)$ are known.

By using the following relations,

$$
\begin{aligned}
\mathbf{g}_{R} & =\mathbf{g}_{d} \mathbf{g}_{e}, \\
\mathbf{g}_{\mathrm{SC}} & =\mathbf{g}_{\mathrm{RB}} \mathbf{g}_{R}, \\
\boldsymbol{\xi}_{R} & =\boldsymbol{\xi}_{e}+\operatorname{Ad}_{\left(\mathbf{g}_{e}\right)^{-1}} \boldsymbol{\xi}_{d}, \\
\boldsymbol{\xi}_{\mathrm{SC}} & =\boldsymbol{\xi}_{R}+\operatorname{Ad}_{\left(\mathrm{g}_{R}\right)^{-1}} \boldsymbol{\xi}_{\mathrm{RB}},
\end{aligned}
$$

(38) and (41) can form complete equations for the tracking error $\left(\mathbf{g}_{e}, \boldsymbol{\xi}_{e}\right)$ of the 6 -DOF relative motion. The goal of the controller is to achieve $\mathbf{g}_{e} \rightarrow \mathbf{I}_{4 \times 4}$ and $\boldsymbol{\xi}_{e} \rightarrow \mathbf{0}$, where $\mathbf{I}_{4 \times 4}$ is the $4 \times 4$ identity matrix.

3.2. Tracking Error in Exponential Coordinates of SE(3). By using exponential coordinates of $\mathrm{SE}(3)$, the tracking error in configuration space of the orbit-attitude motion can be denoted by

$$
\left(\boldsymbol{\eta}_{e}\right)^{\vee}=\log \left(\mathbf{g}_{e}\right)=\log \left(\left[\begin{array}{cc}
\mathbf{A}_{e} & \mathbf{r}_{e} \\
\mathbf{0} & 1
\end{array}\right]\right) \in \operatorname{se}(3),
$$

where the vector of exponential coordinates $\boldsymbol{\eta}_{e}$ is denoted by

$$
\boldsymbol{\eta}_{e}=\left[\begin{array}{l}
\boldsymbol{\Theta}_{e} \\
\mathbf{b}_{e}
\end{array}\right] \in \mathbb{R}^{6},
$$


and the corresponding element of the Lie algebra se(3) is denoted by

$$
\left(\boldsymbol{\eta}_{e}\right)^{\vee}=\left[\begin{array}{cc}
\left(\boldsymbol{\Theta}_{e}\right)^{\times} & \mathbf{b}_{e} \\
\mathbf{0} & 0
\end{array}\right] \in \operatorname{se}(3) .
$$

The specific expressions of the logarithm map log : $\mathrm{SE}(3) \rightarrow \operatorname{se}(3)$ are given by

$$
\begin{aligned}
\left(\Theta_{e}\right)^{\times} & =\frac{\phi}{2 \sin \phi}\left[\mathbf{A}_{e}-\mathbf{A}_{e}^{T}\right], \\
\mathbf{b}_{e} & =\mathbf{A}^{-1}\left(\Theta_{e}\right) \mathbf{r}_{e},
\end{aligned}
$$

where $\phi$ satisfies $\cos \phi=(1 / 2)\left[\operatorname{tr}\left(\mathbf{A}_{e}\right)-1\right], \phi<\pi$, and

$$
\begin{aligned}
\mathbf{A}^{-1}\left(\Theta_{e}\right)= & \mathbf{I}_{3 \times 3}-\frac{1}{2}\left(\Theta_{e}\right)^{\times} \\
& +\left(\frac{1}{\phi^{2}}-\frac{1+\cos \phi}{2 \phi \sin \phi}\right)\left(\Theta_{e}\right)^{\times}\left(\Theta_{e}\right)^{\times} .
\end{aligned}
$$

Actually, $\boldsymbol{\Theta}_{e}=\phi \mathbf{n}$ is the principal rotation vector of attitude matrix $\mathbf{A}_{e} ; \phi=\left|\Theta_{e}\right|$ and unit vector $\mathbf{n}$ are the principal rotation angle and axis, respectively. The logarithm map log : $\mathrm{SE}(3) \rightarrow \mathrm{se}(3)$ is bijective when the principal rotation angle of $\mathbf{A}_{e}$ is less than a $\pi$ radian, that is, $\phi<\pi$, but it is not uniquely defined when $\phi$ is exactly a $\pi$ radian [15].

The kinematics of tracking error of the spacecraft's relative motion (38) can be rewritten in terms of exponential coordinates $\boldsymbol{\eta}_{e}$ :

$$
\dot{\boldsymbol{\eta}}_{e}=\mathbf{G}\left(\boldsymbol{\eta}_{e}\right) \boldsymbol{\xi}_{e} .
$$

The kinematical matrix $\mathbf{G}\left(\boldsymbol{\eta}_{e}\right)$ is given by [28]

$$
\begin{aligned}
\mathbf{G}\left(\boldsymbol{\eta}_{e}\right)= & \mathbf{I}_{6 \times 6}+\frac{1}{2} \operatorname{ad}_{\boldsymbol{\eta}_{e}}+A(\phi)\left(\operatorname{ad}_{\boldsymbol{\eta}_{e}}\right)^{2} \\
& +B(\phi)\left(\operatorname{ad}_{\boldsymbol{\eta}_{e}}\right)^{4},
\end{aligned}
$$

where $\mathbf{I}_{6 \times 6}$ is the $6 \times 6$ identity matrix:

$$
\begin{aligned}
\operatorname{ad}_{\boldsymbol{\eta}_{e}} & =\left[\begin{array}{cc}
\left(\boldsymbol{\Theta}_{e}\right)^{\times} & \mathbf{0} \\
\left(\mathbf{b}_{e}\right)^{\times} & \left(\boldsymbol{\Theta}_{e}\right)^{\times}
\end{array}\right], \\
A(\phi) & =\frac{2}{\phi^{2}}[1-\alpha(\phi)]+\frac{1}{2 \phi^{2}}[\alpha(\phi)-\beta(\phi)], \\
B(\phi) & =\frac{1}{\phi^{4}}[1-\alpha(\phi)]+\frac{1}{2 \phi^{4}}[\alpha(\phi)-\beta(\phi)], \\
\alpha(\phi) & =\frac{\phi}{2} \cot \left(\frac{\phi}{2}\right), \\
\beta(\phi) & =\left[\frac{(\phi / 2)}{\sin (\phi / 2)}\right]^{2} .
\end{aligned}
$$

Equations (48) and (41) can form complete equations of motion for the tracking error of spacecraft's 6-DOF relative motion in terms of exponential coordinates $\left(\boldsymbol{\eta}_{e}, \boldsymbol{\xi}_{e}\right)$. The goal of the controller is to achieve $\boldsymbol{\eta}_{e} \rightarrow \mathbf{0}$ and $\boldsymbol{\xi}_{e} \rightarrow \mathbf{0}$.
3.3. Dynamics of Tracking Error as a Second-Order System. In equations of motion (48) and (41), exponential coordinates of SE(3) are used only on the kinematical level. That is, (48) has only given the relation between the first-order time derivative of exponential coordinates and the velocity. In the following, we will extend exponential coordinates to the dynamical level, by giving the relation between the second-order time derivative of exponential coordinates and the acceleration. This extension is an important improvement compared with earlier works by Lee et al. $[6,15,16]$.

By taking time derivative of both sides of the kinematics of tracking error (48), we can have the second-order derivative of exponential coordinates:

$$
\ddot{\boldsymbol{\eta}}_{e}=\mathbf{G}\left(\boldsymbol{\eta}_{e}\right) \dot{\boldsymbol{\xi}}_{e}+\dot{\mathbf{G}}\left(\boldsymbol{\eta}_{e}\right) \boldsymbol{\xi}_{e},
$$

where, according to (49), $\dot{\mathbf{G}}\left(\boldsymbol{\eta}_{e}\right)$ can be obtained as

$$
\begin{aligned}
& \dot{\mathbf{G}}\left(\boldsymbol{\eta}_{e}\right)=\frac{1}{2} \mathrm{ad}_{\boldsymbol{\eta}_{e}}+\dot{A}(\phi)\left(\mathrm{ad}_{\boldsymbol{\eta}_{e}}\right)^{2}+A(\phi)\left(\operatorname{ad}_{\boldsymbol{\eta}_{e}} \mathrm{ad}_{\boldsymbol{\eta}_{e}}\right. \\
& \left.+\operatorname{ad}_{\boldsymbol{\eta}_{e}} \dot{a d}_{\boldsymbol{\eta}_{e}}\right)+\dot{B}(\phi)\left(\operatorname{ad}_{\boldsymbol{\eta}_{e}}\right)^{4}+B(\phi)\left[\operatorname{ad}_{\boldsymbol{\eta}_{e}}\left(\operatorname{ad}_{\boldsymbol{\eta}_{e}}\right)^{3}\right. \\
& +\operatorname{ad}_{\boldsymbol{\eta}_{e}} \operatorname{ad}_{\boldsymbol{\eta}_{e}}\left(\operatorname{ad}_{\boldsymbol{\eta}_{e}}\right)^{2}+\left(\operatorname{ad}_{\boldsymbol{\eta}_{e}}\right)^{2} \operatorname{ad}_{\boldsymbol{\eta}_{e}} \operatorname{ad}_{\boldsymbol{\eta}_{e}} \\
& \left.+\left(\operatorname{ad}_{\eta_{e}}\right)^{3} \mathrm{ad}_{\eta_{e}}\right] \text {. }
\end{aligned}
$$

According to (50), ad $\dot{\eta}_{\eta_{e}}$ in (56) is given by

$$
\mathrm{ad}_{\eta_{e}}=\left[\begin{array}{cc}
\left(\dot{\boldsymbol{\Theta}}_{e}\right)^{\times} & 0 \\
\left(\dot{\mathbf{b}}_{e}\right)^{\times} & \left(\dot{\boldsymbol{\Theta}}_{e}\right)^{\times}
\end{array}\right],
$$

where $\dot{\Theta}_{e}$ and $\dot{\mathbf{b}}_{e}$ are given by (48). By using (51) and (52), $\dot{A}(\phi)$ and $\dot{B}(\phi)$ in (56) can be derived as

$$
\begin{aligned}
& \dot{A}(\phi)=\frac{d A}{d \phi} \frac{d \phi}{d t} \\
& =\frac{1}{\phi^{3}}\left[\frac{3}{2} \alpha(\phi)+\frac{3}{2} \beta(\phi)+\alpha(\phi) \beta(\phi)-4\right] \frac{\Theta_{e} \cdot \dot{\Theta}_{e}}{\phi}, \\
& \dot{B}(\phi)=\frac{d B}{d \phi} \frac{d \phi}{d t} \\
& =\frac{1}{\phi^{5}}\left[\frac{3}{2} \alpha(\phi)+\frac{3}{2} \beta(\phi)+\alpha(\phi) \beta(\phi)-4\right] \frac{\Theta_{e} \cdot \dot{\Theta}_{e}}{\phi} .
\end{aligned}
$$

Then, (55) and (41) can form a second-order equation of tracking error of the 6-DOF relative motion in terms of exponential coordinates $\left(\boldsymbol{\eta}_{e}, \boldsymbol{\xi}_{e}\right)$ :

$$
\ddot{\boldsymbol{\eta}}_{e}=\mathbf{G}\left(\boldsymbol{\eta}_{e}\right) \dot{\boldsymbol{\xi}}_{e}+\dot{\mathbf{G}}\left(\boldsymbol{\eta}_{e}\right) \boldsymbol{\xi}_{e},
$$

where $\dot{\boldsymbol{\xi}}_{e}, \mathbf{G}\left(\boldsymbol{\eta}_{e}\right)$, and $\dot{\mathbf{G}}\left(\boldsymbol{\eta}_{e}\right)$ are given by (41), (49), and (56), respectively.

3.4. Integrated Tracking Control Law with Almost Global Convergence. Based on the dynamics of tracking error of 
the 6-DOF relative motion, appropriate control theories can be applied, and integrated tracking control laws can be designed. To show the application potential of our integrated 6-DOF dynamical modeling (59) in terms of exponential coordinates, a simple integrated tracking control law will be designed for a demonstration purpose.

Since the exponential coordinates are not uniquely defined when the rotation angle is exactly a $\pi$ radian, the controllers can work with almost all initial tracking errors, except those with a rotation angle of a $\pi$ radian. Because the set of such initial states is an embedded lower-dimensional subspace of the state space, the tracking control law designed in terms of exponential coordinates can achieve almost global convergence and then is applicable to space missions and operations in practice.

By using a simple feedback scheme, the continuous tracking control law $\boldsymbol{\tau}_{\mathrm{SC}}^{c}$ for the integrated orbit-attitude motion of spacecraft can be designed as

$$
\begin{aligned}
\boldsymbol{\tau}_{\mathrm{SC}}^{c} & =-\operatorname{ad}_{\boldsymbol{\xi}_{\mathrm{SC}}}^{*} \rrbracket_{\mathrm{SC}} \boldsymbol{\xi}_{\mathrm{SC}}-\boldsymbol{\tau}_{\mathrm{SC}}^{n}-\rrbracket_{\mathrm{SC}} \mathbf{G}^{-1}\left(\boldsymbol{\eta}_{e}\right)\left[\dot{\mathbf{G}}\left(\boldsymbol{\eta}_{e}\right) \boldsymbol{\xi}_{e}\right. \\
& \left.+\mathbf{K}_{d} \dot{\boldsymbol{\eta}}_{e}+\mathbf{K}_{p} \boldsymbol{\eta}_{e}\right]-\mathbb{\square}_{\mathrm{SC}}\left\{\operatorname{ad}_{\xi_{e}} \operatorname{Ad}_{\left(\mathbf{g}_{e}\right)^{-1}} \boldsymbol{\xi}_{d}-\operatorname{Ad}_{\left(\mathbf{g}_{e}\right)^{-1}} \dot{\boldsymbol{\xi}}_{d}\right. \\
& \left.+\operatorname{ad}_{\xi_{R}} \operatorname{Ad}_{\left(\mathbf{g}_{R}\right)^{-1}} \boldsymbol{\xi}_{\mathrm{RB}}-\operatorname{Ad}_{\left(\mathbf{g}_{R}\right)^{-1}} \dot{\boldsymbol{\xi}}_{\mathrm{RB}}\right\}
\end{aligned}
$$

where the control gains $\mathbf{K}_{d}$ and $\mathbf{K}_{p}$ can be tuned to achieve desired stability and dynamic performance of the closed-loop system.

The dynamics of closed-loop system describing tracking error of the 6-DOF relative motion is obtained by substituting the control law (60) into the second-order equation of tracking error (59):

$$
\ddot{\boldsymbol{\eta}}_{e}+\mathbf{K}_{d} \dot{\boldsymbol{\eta}}_{e}+\mathbf{K}_{p} \boldsymbol{\eta}_{e}=\mathbf{0}
$$

which is a homogeneous linear differential equation with constant coefficients. By choosing appropriate control gains $\mathbf{K}_{d}$ and $\mathbf{K}_{p}$ in the control law (60), the tracking error in configuration space $\boldsymbol{\eta}_{e}$ and its derivative $\dot{\boldsymbol{\eta}}_{e}$ will both converge to $\mathbf{0}$ asymptotically, and, according to (48), the tracking error in velocity $\boldsymbol{\xi}_{e}$ will also converge to $\mathbf{0}$ asymptotically.

Therefore, with the feedback control law (60), the spacecraft's 6-DOF relative motion will converge to the desired trajectory asymptotically from almost all initial tracking errors, except those with a rotation angle of a $\pi$ radian. Thus, the control law (60) is an integrated asymptotic tracking control law with almost global convergence.

\section{Body-Fixed Orbit-Attitude Hovering over an Asteroid}

In this section, we will apply integrated dynamical modeling and tracking control law for the spacecraft's 6-DOF motion obtained above to the body-fixed orbit-attitude hovering over an asteroid.

The body-fixed orbit-attitude hovering means that both the position and attitude of the spacecraft are kept to be stationary in the asteroid body-fixed frame [29]. The orbitattitude hovering was modeled also in an integrated manner by Wang and $\mathrm{Xu}$ [29] by using the theory of noncanonical Hamiltonian system within the framework of gravitationally coupled orbit-attitude dynamics, in which the spacecraft was considered as a rigid body. Wang and Xu [29] have proposed a noncanonical Hamiltonian structure-based feedback control law. Here, we will use the asymptotic tracking control law designed in Section 3.4 to achieve the orbit-attitude hovering and make comparisons with the controller in Wang and Xu [29].

4.1. System Description. It is assumed that the center of mass of the asteroid, that is, the reference body in earlier parts of the paper, is stationary in the inertial space, and the asteroid is rotating with a constant angular velocity $\omega_{T}$ around its maximum-moment principal axis, which is assumed to be $\mathbf{w}$ axis without loss of generality. That is to say, the external torque and force acting on the asteroid, $\boldsymbol{\tau}_{\mathrm{RB}}$, are zeros. Thus, the 6-DOF dynamics of the reference body can be simplified as

$$
\begin{aligned}
\mathbf{g}_{\mathrm{RB} 0} & =\mathbf{I}_{4 \times 4}, \\
\boldsymbol{\xi}_{\mathrm{RB}} & \equiv\left[0,0, \omega_{T}, 0,0,0\right]^{T}, \\
\dot{\boldsymbol{\xi}}_{\mathrm{RB}} & =\mathbf{0} .
\end{aligned}
$$

To make comparisons with the controller in Wang and $\mathrm{Xu}$ [29], a similar mission scenario is chosen in the following. The gravity field of the asteroid is approximated by a second degree and order-gravity field with harmonics $C_{20}$ and $C_{22}$. The parameters of the asteroid are chosen to be the same as in Wang and $\mathrm{Xu}[29]$ :

$$
\begin{aligned}
\mu & =94 \mathrm{~m}^{3} / \mathrm{s}^{2}, \\
C_{20} & =-0.1, \\
C_{22} & =0.04, \\
a_{e} & =400 \mathrm{~m}, \\
\omega_{T} & =2.9089 \times 10^{-4} \mathrm{~s}^{-1},
\end{aligned}
$$

where $\mu=G m_{\mathrm{RB}}, G$ is the gravitational constant, and $a_{e}$ is the mean equatorial radius of the asteroid.

Since the spacecraft is in the close proximity of the asteroid and is assumed to have a low area-to-mass ratio, perturbations of solar gravity and SRP are negligible. Thus, only the gravitational torque and force by asteroid are considered in the environmental torque and force $\boldsymbol{\tau}_{\mathrm{SC}}^{n}$. That is to say, $\boldsymbol{\tau}_{\mathrm{SC}}^{n}$ depends only on the relative configuration of spacecraft with respect to the asteroid:

$$
\boldsymbol{\tau}_{\mathrm{SC}}^{n}=\boldsymbol{\tau}_{\mathrm{SC}}^{n}\left(\mathbf{g}_{R}\right)
$$

The explicit formulation of $\boldsymbol{\tau}_{\mathrm{SC}}^{n}\left(\mathbf{g}_{R}\right)$ is given by [19]

$$
\begin{aligned}
& \mathbf{T}_{\mathrm{SC}}^{n}=\frac{3 \mu}{R_{R}^{5}} \mathbf{R}_{R} \times \mathbf{I}_{\mathrm{SC}} \mathbf{R}_{R}, \\
& \mathbf{F}_{\mathrm{SC}}^{n}=-\frac{\mu m_{\mathrm{SC}}}{R_{R}^{2}} \overline{\mathbf{R}}_{R}+\frac{3 \mu}{2 R_{R}^{4}}\left\{\left[5 \overline{\mathbf{R}}_{R}^{T} \mathbf{I}_{\mathrm{SC}} \overline{\mathbf{R}}_{R}-\operatorname{tr}\left(\mathbf{I}_{\mathrm{SC}}\right)\right.\right.
\end{aligned}
$$




$$
\begin{aligned}
& +\tau_{0} m_{\mathrm{SC}}\left(1-5\left(\boldsymbol{\gamma}_{R} \cdot \overline{\mathbf{R}}_{R}\right)^{2}\right) \\
& \left.-10 \tau_{2} m_{\mathrm{SC}}\left(\left(\boldsymbol{\alpha}_{R} \cdot \overline{\mathbf{R}}_{R}\right)^{2}-\left(\boldsymbol{\beta}_{R} \cdot \overline{\mathbf{R}}_{R}\right)^{2}\right)\right] \overline{\mathbf{R}}_{R} \\
& -2 \mathbf{I}_{\mathrm{SC}} \overline{\mathbf{R}}_{R}+2 \tau_{0} m_{\mathrm{SC}}\left(\boldsymbol{\gamma}_{R} \cdot \overline{\mathbf{R}}_{R}\right) \boldsymbol{\gamma}_{R} \\
& \left.+4 \tau_{2} m_{\mathrm{SC}}\left(\left(\boldsymbol{\alpha}_{R} \cdot \overline{\mathbf{R}}_{R}\right) \boldsymbol{\alpha}_{R}-\left(\boldsymbol{\beta}_{R} \cdot \overline{\mathbf{R}}_{R}\right) \boldsymbol{\beta}_{R}\right)\right\},
\end{aligned}
$$

where $\mathbf{R}_{R}=\mathbf{A}_{R}^{-1} \mathbf{r}_{R}$ is the relative position of the spacecraft with respect to the asteroid expressed in the body-fixed frame of spacecraft $S_{\mathrm{SC}}, \overline{\mathbf{R}}_{R}$ is the unit vector along $\mathbf{R}_{R}, \tau_{0}=$ $a_{e}^{2} C_{20}$, and $\tau_{2}=a_{e}^{2} C_{22}$. In the environmental force $\mathbf{F}_{\mathrm{SC}}^{n}$, the perturbation caused by the gravitational orbit-attitude coupling of the spacecraft has been included [30].

The parameters of spacecraft are chosen as

$$
\begin{aligned}
m_{\mathrm{SC}} & =1 \times 10^{3} \mathrm{~kg}, \\
\mathbf{I}_{\mathrm{SC}} & =\left[\begin{array}{ccc}
2 & 0 & 0 \\
0 & 1 & 0 \\
0 & 0 & 1.6
\end{array}\right] \times 10^{3} \mathrm{~kg} \cdot \mathrm{m}^{2} .
\end{aligned}
$$

The hovering position-attitude $\mathbf{g}_{\mathrm{RH}}$, that is, the relative configuration of spacecraft at hovering, is chosen to be the same as in Wang and $\mathrm{Xu}[29]$ :

$$
\mathbf{g}_{\mathrm{RH}}=\left[\begin{array}{cc}
\mathbf{A}_{\mathrm{RH}} & \mathbf{r}_{\mathrm{RH}} \\
\mathbf{0} & 1
\end{array}\right] \in \mathrm{SE}(3),
$$

$$
\begin{aligned}
& \mathbf{A}_{R 0}=\mathbf{A}_{\mathrm{RH}} \mathbf{L}, \\
& \mathbf{r}_{R 0}=\mathbf{r}_{\mathrm{RH}}+[200,100,250]^{T} \mathrm{~m} . \\
& \boldsymbol{\xi}_{R 0}=\left[\begin{array}{l}
\boldsymbol{\Omega}_{R 0} \\
\mathbf{V}_{R 0}
\end{array}\right]=\left[-0.06 \mathrm{~s}^{-1}, 0.05 \mathrm{~s}^{-1}, 0.09 \mathrm{~s}^{-1},\right. \\
& -1 \mathrm{~m} / \mathrm{s}, 2 \mathrm{~m} / \mathrm{s}, 1.5 \mathrm{~m} / \mathrm{s}]^{T} \text {. }
\end{aligned}
$$$$
\mathbf{L}=\left[\begin{array}{ccc}
\cos \left(\frac{\pi}{9}\right) & -\sin \left(\frac{\pi}{9}\right) & 0 \\
\sin \left(\frac{\pi}{9}\right) & \cos \left(\frac{\pi}{9}\right) & 0 \\
0 & 0 & 1
\end{array}\right]\left[\begin{array}{ccc}
\cos \left(-\frac{\pi}{20}\right) & 0 & \sin \left(-\frac{\pi}{20}\right) \\
0 & 1 & 0 \\
-\sin \left(-\frac{\pi}{20}\right) & 0 & \cos \left(-\frac{\pi}{20}\right)
\end{array}\right]\left[\begin{array}{ccc}
1 & 0 & 0 \\
0 & \cos \left(\frac{\pi}{18}\right) & -\sin \left(\frac{\pi}{18}\right) \\
0 & \sin \left(\frac{\pi}{18}\right) & \cos \left(\frac{\pi}{18}\right)
\end{array}\right],
$$

The initial relative velocity of spacecraft with respect to the asteroid $\xi_{R 0}$ is chosen as

In simulations, absolute motions of the spacecraft and asteroid in the inertial space will be simulated separately. The where

$$
\begin{aligned}
\mathbf{A}_{\mathrm{RH}} & =\left[\boldsymbol{\alpha}_{\mathrm{RH}}, \boldsymbol{\beta}_{\mathrm{RH}}, \boldsymbol{\gamma}_{\mathrm{RH}}\right]^{T}, \\
\mathbf{r}_{\mathrm{RH}} & =\mathbf{A}_{\mathrm{RH}} \mathbf{R}_{\mathrm{RH}}, \\
\boldsymbol{\alpha}_{\mathrm{RH}} & =[0.9659,0,-0.2588]^{T}, \\
\boldsymbol{\beta}_{\mathrm{RH}} & =[0.067,0.9659,0.25]^{T}, \\
\boldsymbol{\gamma}_{\mathrm{RH}} & =[0.25,-0.2588,0.933]^{T}, \\
\mathbf{R}_{\mathrm{RH}} & =500[0.9798,0,0.2]^{T} \mathrm{~m} .
\end{aligned}
$$

In the simulation, the desired trajectory $\left(\mathbf{g}_{d}(t), \boldsymbol{\xi}_{d}(t)\right.$, $\left.\dot{\boldsymbol{\xi}}_{d}(t)\right)$ is simply chosen as the desired hovering positionattitude:

$$
\begin{aligned}
& \mathbf{g}_{d}(t) \equiv \mathbf{g}_{\mathrm{RH}}, \\
& \boldsymbol{\xi}_{d}(t) \equiv \mathbf{0}, \\
& \dot{\boldsymbol{\xi}}_{d}(t) \equiv \mathbf{0} .
\end{aligned}
$$

A better reference trajectory connecting the initial positionattitude and the hovering position-attitude, which is easier to track, can be designed in the future through studies on the guidance on $\mathrm{SE}(3)$, which is not the main concern of this paper.

The initial relative configuration of spacecraft with respect to the asteroid $\mathbf{g}_{R 0}$ is chosen as

$$
\mathbf{g}_{R 0}=\left[\begin{array}{cc}
\mathbf{A}_{R 0} & \mathbf{r}_{R 0} \\
\mathbf{0} & 1
\end{array}\right] \in \mathrm{SE}(3),
$$

where relative motion of spacecraft and its tracking error that appear in the control law will be calculated by using absolute motions and the desired trajectory. With this approach, not only the control law but also the dynamical modeling method can be verified.

To initiate the simulation of spacecraft's absolute motion, the initial configuration of spacecraft in the inertial space can be calculated by

$$
\mathbf{g}_{\mathrm{SC} 0}=\mathbf{g}_{\mathrm{RB} 0} \mathbf{g}_{R 0},
$$



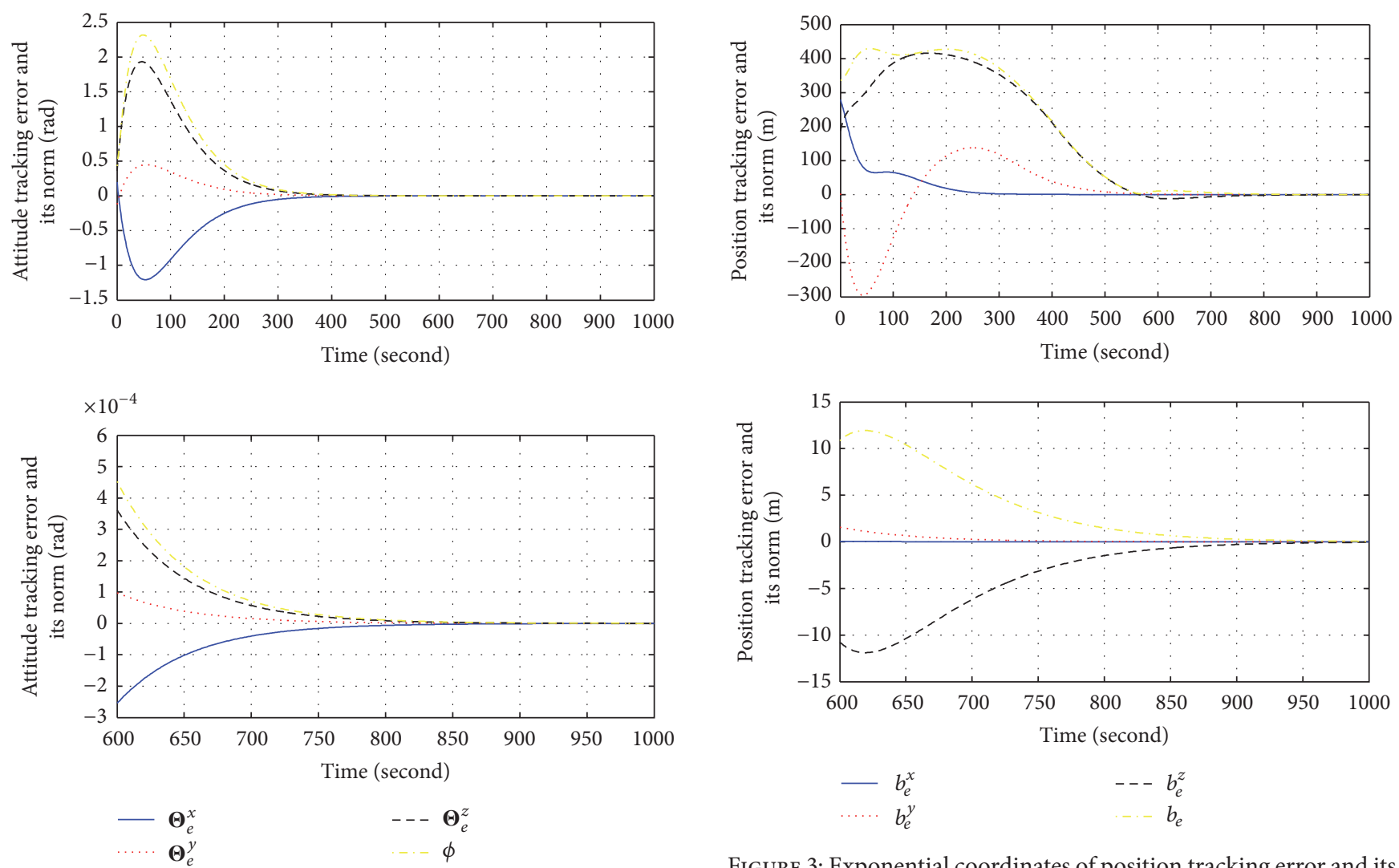

FIGURE 2: Exponential coordinates of attitude tracking error and its norm.

and the initial velocity of spacecraft in the inertial space can be calculated by

$$
\xi_{\mathrm{SC} 0}=\xi_{R 0}+\operatorname{Ad}_{\left(\mathrm{g}_{R 0}\right)^{-1}} \boldsymbol{\xi}_{\mathrm{RB} 0} .
$$

The control gains $\mathbf{K}_{d}$ and $\mathbf{K}_{p}$ in the control law (60) are chosen as

$$
\begin{aligned}
& \mathbf{K}_{d}=0.04 \mathbf{I}_{6 \times 6}, \\
& \mathbf{K}_{p}=4 \times 10^{-4} \mathbf{I}_{6 \times 6} .
\end{aligned}
$$

The maximum control torque and force that can be provided by the spacecraft's control system are set to be $10 \mathrm{~N} \cdot \mathrm{m}$ and $10 \mathrm{~N}$ for each axis, respectively.

4.2. Numerical Simulation Results. The exponential coordinates of the position-attitude tracking error $\boldsymbol{\eta}_{e}=\left[\Theta_{e}^{T}, \mathbf{b}_{e}^{T}\right]^{T}$ and their norms are shown in Figures 2 and 3, respectively, and the tracking errors of the spacecraft's angular velocity and velocity $\boldsymbol{\xi}_{e}=\left[\boldsymbol{\Omega}_{e}^{T}, \mathbf{V}_{e}^{T}\right]^{T}$ are shown in Figures 4 and 5, respectively. It can be seen that the motion of spacecraft converges to the hovering position-attitude and keeps motionless relative to the asteroid after about 900 seconds. The attitude motion of spacecraft has an error of about 0.026 degrees and 5 $\times 10^{-4}$ degree/s within 600 seconds and an error of about $1.5 \times$ $10^{-5}$ degrees and $3 \times 10^{-7}$ degree/s within 1000 seconds, while the orbital motion has an error of about $10 \mathrm{~m}$ and $0.15 \mathrm{~m} / \mathrm{s}$

FIGURE 3: Exponential coordinates of position tracking error and its norm.
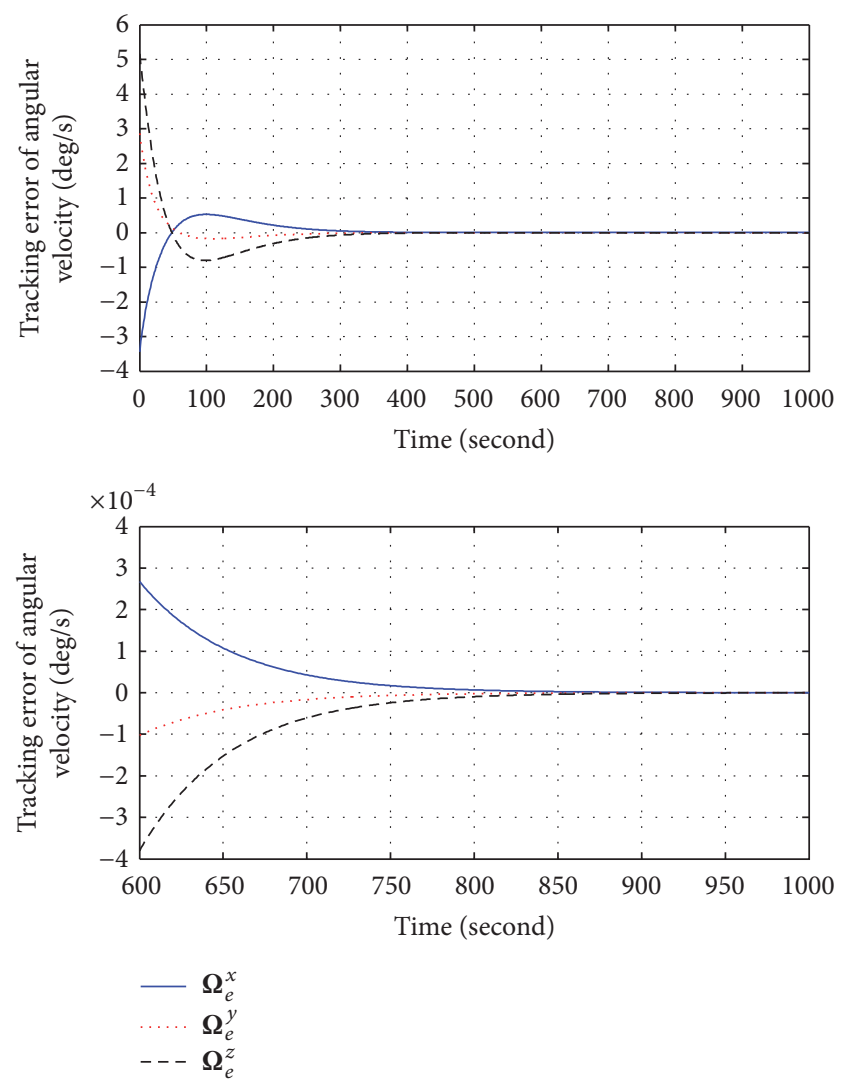

FigURE 4: Tracking error of the angular velocity of spacecraft. 

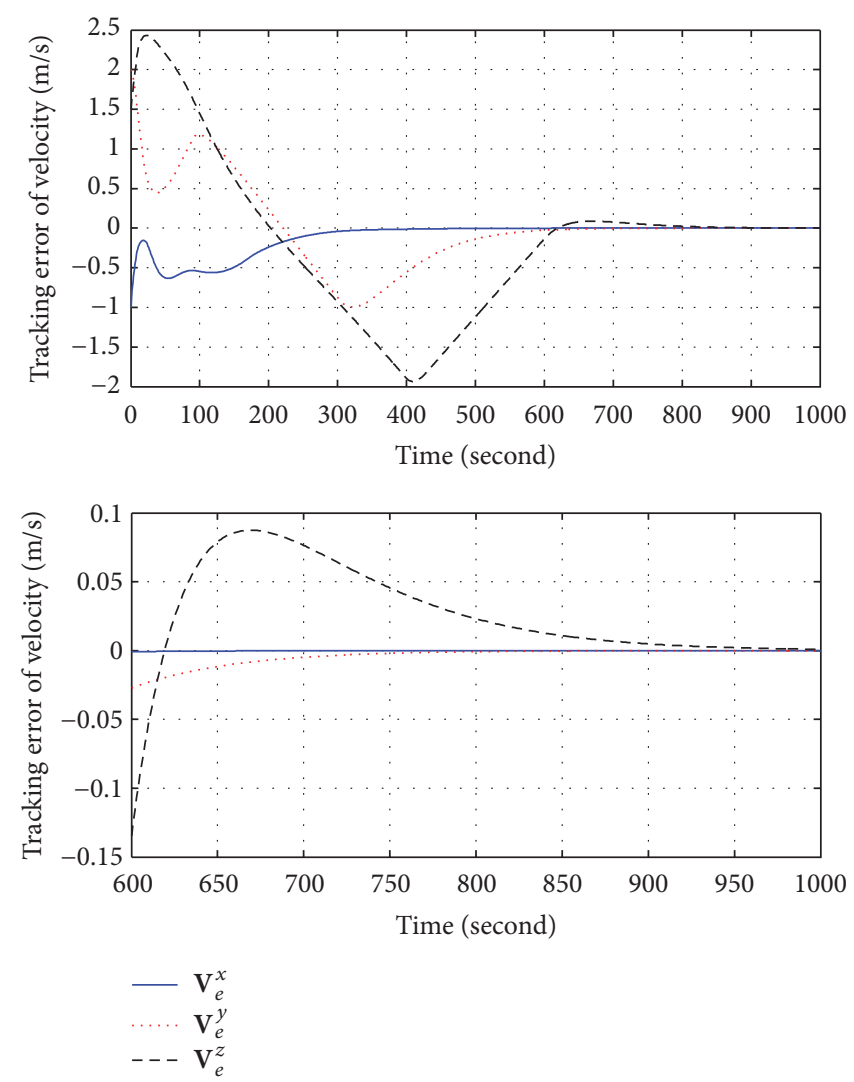

FIGURE 5: Tracking error of the velocity of spacecraft.

within 600 seconds and an error of about $0.05 \mathrm{~m}$ and $1 \times$ $10^{-3} \mathrm{~m} / \mathrm{s}$ within 1000 seconds. The attitude motion has a faster converge than the orbital motion.

The trajectory of spacecraft in the body-fixed frame of asteroid in Figure 6 shows that it does not approach the hovering position directly due to the initial velocity error. In the figure, the hovering position is denoted by the star $(*)$, the initial position of spacecraft is denoted by the circle $(O)$, and the final position of spacecraft is denoted by the pentagram $(\xi)$. Notice that the star $(*)$ is overlapped with the pentagram (ఓ) and cannot be distinguished.

The components of control torque and force $\mathbf{T}_{\mathrm{SC}}^{c}$ and $\mathbf{F}_{\mathrm{SC}}^{c}$, both expressed in the spacecraft body-fixed frame $S_{\mathrm{SC}}$, are shown in Figures 7 and 8, respectively. The control torque is within the spacecraft's control capacity during the whole trajectory, whereas the thrust saturation of the control force occurs on all three axes before 600 seconds. Although thrust saturation occurs for the control force, the controller is still effective.

As the spacecraft is approaching to the hovering configuration, the control torque and force are converging to values that are needed to balance the gravitational and centrifugal torque and force at hovering position-attitude. Since the gravity gradient torque $\mathrm{T}_{\mathrm{SC}}^{n}$ is quite small, the control torque $\mathrm{T}_{\mathrm{SC}}^{c}$ converges to nearly zero.

Compared with the hovering controller in Wang and $\mathrm{Xu}$ [29], the controller proposed in this paper has not utilized the Hamiltonian structure of the system. On one hand, the controller in this paper (60) is much more complicated than that in Wang and $\mathrm{Xu}$ [29], which is consisted of two potential shapings and one energy dissipation. However, on the other hand, the closed-loop system in this paper is a linear system and converges much faster than that in Wang and $\mathrm{Xu}$ [29], which is a noncanonical Hamiltonian system with energy dissipation, oscillating around the hovering position-attitude for a long duration before the final convergence.

\section{Conclusions}

The integrated 6-DOF orbit-attitude dynamical modeling and controller design for the relative motion of spacecraft with respect to a reference body have been studied in the framework of geometric mechanics in the present paper. The configuration and velocity of the relative motion have been represented by the Lie group $\mathrm{SE}(3)$ and also its exponential coordinates. By using exponential coordinates of SE(3) on the dynamical level, the dynamics of tracking error have been formulated as a second-order system with a quite compact form. A simple asymptotic tracking control law with almost global convergence was designed for a demonstration purpose. Both the integrated dynamical modeling and control law were verified in an asteroid hovering mission scenario. To verify the dynamical modeling method, individual motions of the spacecraft and asteroid in the inertial space have been simulated, rather than the equations of motion describing the relative dynamics. 

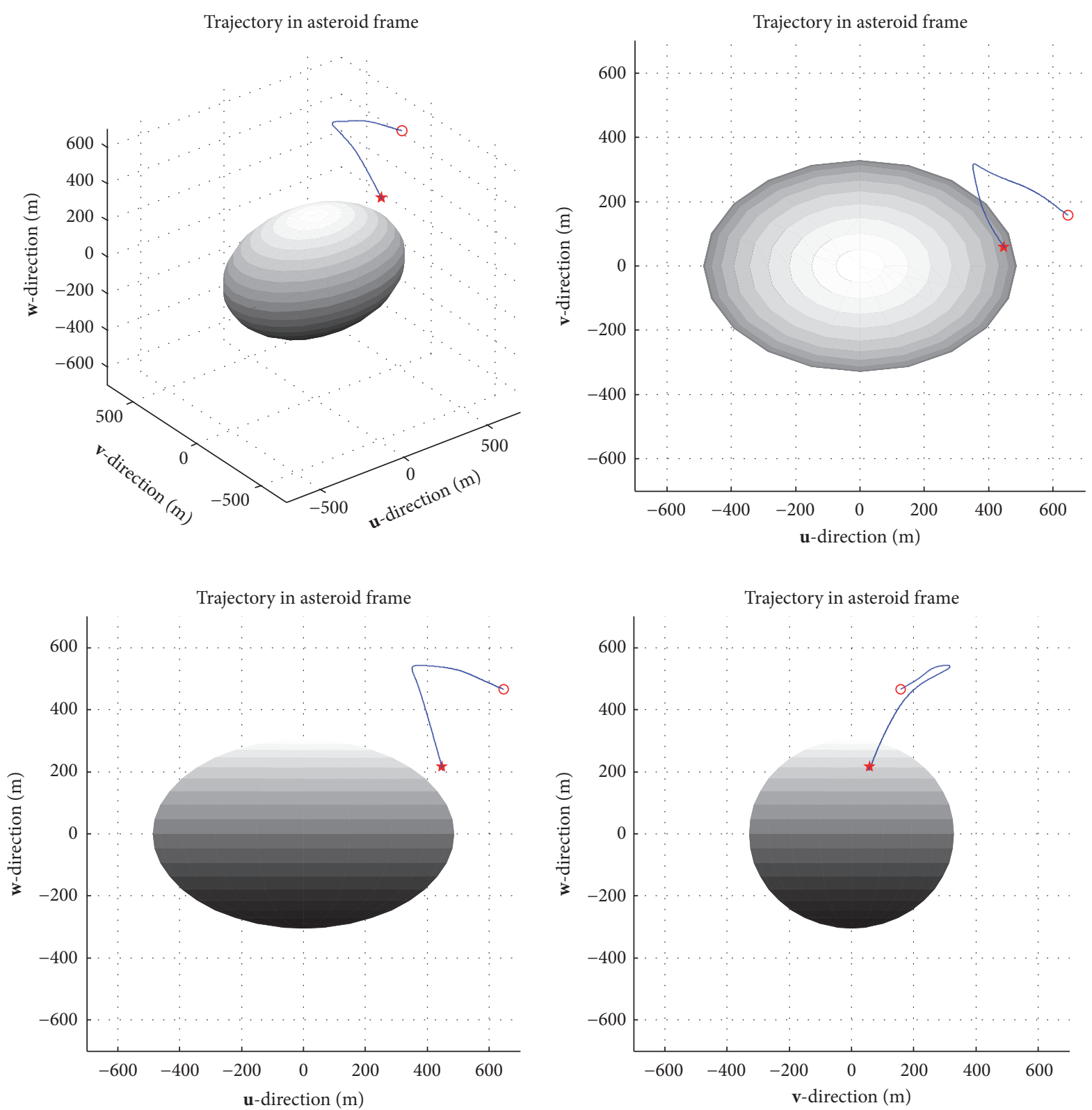

FIGURE 6: Trajectory of the spacecraft in the asteroid body-fixed frame.

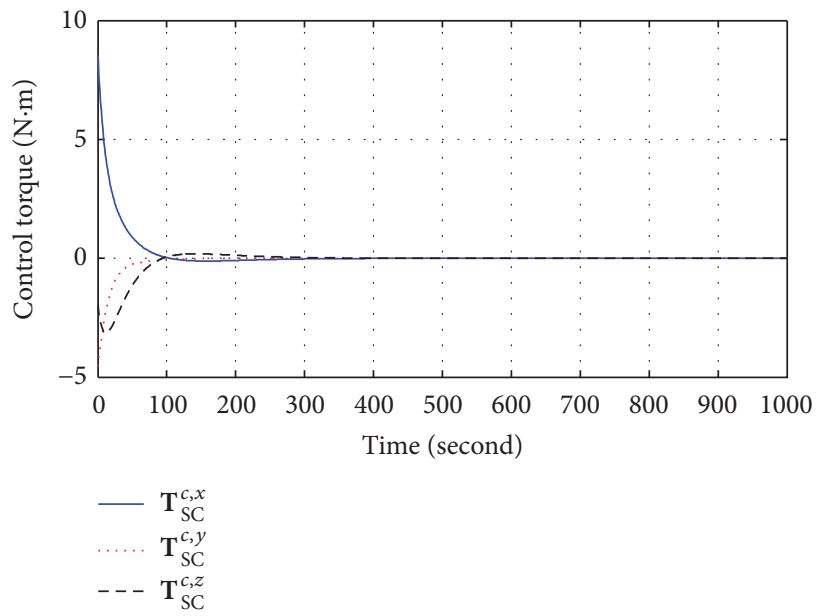

Figure 7: Components of control torque on the three axes of the spacecraft. 


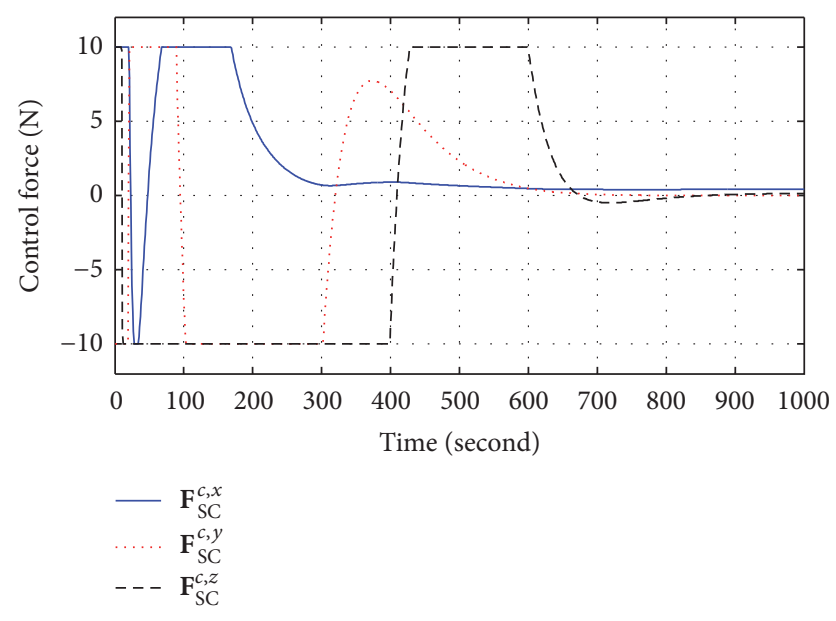

FIGURE 8: Components of control force on the three axes of the spacecraft.

\section{Conflicts of Interest}

The authors declare that they have no conflicts of interest.

\section{Acknowledgments}

This work has been supported by the National Natural Science Foundation of China under Grants 11432001 and 11602009 and the Fundamental Research Funds for the Central Universities.

\section{References}

[1] S. Segal and P. Gurfil, "Effect of kinematic rotation-translation coupling on relative spacecraft translational dynamics," Journal of Guidance, Control, and Dynamics, vol. 32, no. 3, pp. 10451050, 2009.

[2] J. Wang and Z. Sun, "6-DOF robust adaptive terminal sliding mode control for spacecraft formation flying," Acta Astronautica, vol. 73, pp. 76-87, 2012.

[3] J. Wu, K. Liu, and D. Han, "Adaptive sliding mode control for six-DOF relative motion of spacecraft with input constraint," Acta Astronautica, vol. 87, pp. 64-76, 2013.

[4] M. Massari and M. Zamaro, "Application of SDRE technique to orbital and attitude control of spacecraft formation flying," Acta Astronautica, vol. 94, no. 1, pp. 409-420, 2014.

[5] Q. Lan, J. Yang, S. Li, and H. Sun, "Finite-time control for 6DOF spacecraft formation flying systems," Journal of Aerospace Engineering, vol. 28, no. 5, Article ID 04014137, 2015.

[6] D. Lee, A. K. Sanyal, and E. A. Butcher, "Asymptotic tracking control for spacecraft formation flying with decentralized collision avoidance," Journal of Guidance, Control, and Dynamics, vol. 38, no. 4, pp. 587-600, 2015.

[7] D. Lee, H. Bang, E. A. Butcher, and A. K. Sanyal, "Kinematically coupled relative spacecraft motion control using the statedependent riccati equation method," Journal of Aerospace Engineering, vol. 28, no. 4, Article ID 4014099, 2015.

[8] A. Sanyal, L. Holguin, and S. P. Viswanathan, "Guidance and control for spacecraft autonomous chasing and close proximity maneuvers," in Proceedings of the 7th IFAC Symposium on Robust Control Design (ROCOND '12), pp. 753-758, 2012.
[9] D. Lee, "Spacecraft coupled tracking maneuver using sliding mode control with input saturation," Journal of Aerospace Engineering, vol. 28, no. 5, Article ID 04014136, 2015.

[10] H. Gui and G. Vukovich, "Dual-quaternion-based adaptive motion tracking of spacecraft with reduced control effort," Nonlinear Dynamics, vol. 83, no. 1-2, pp. 1-18, 2015.

[11] N. Filipe and P. Tsiotras, "Adaptive position and attitude-tracking controller for satellite proximity operations using dual quaternions," Journal of Guidance, Control, and Dynamics, vol. 38, no. 4, pp. 566-577, 2015.

[12] L. Sun and W. Huo, "Robust adaptive control of spacecraft proximity maneuvers under dynamic coupling and uncertainty," Advances in Space Research, vol. 56, no. 10, pp. 2206-2217, 2015.

[13] L. Sun and W. Huo, "Robust adaptive relative position tracking and attitude synchronization for spacecraft rendezvous," Aerospace Science and Technology, vol. 41, pp. 28-35, 2015.

[14] D. Lee and G. Vukovich, "Robust adaptive terminal sliding mode control on SE(3) for autonomous spacecraft rendezvous and docking," Nonlinear Dynamics, vol. 83, no. 4, pp. 2263-2279, 2016.

[15] D. Lee, A. K. Sanyal, E. A. Butcher, and D. J. Scheeres, "Almost global asymptotic tracking control for spacecraft body-fixed hovering over an asteroid," Aerospace Science and Technology, vol. 38, pp. 105-115, 2014.

[16] D. Lee, A. Sanyal, E. Butcher, and D. Scheeres, "Finite-time control for spacecraft body-fixed hovering over an asteroid," IEEE Transactions on Aerospace and Electronic Systems, vol. 51, no. 1, pp. 506-520, 2015.

[17] D. Lee and G. Vukovich, "Adaptive sliding mode control for spacecraft body-fixed hovering in the proximity of an asteroid," Aerospace Science and Technology, vol. 46, pp. 471-483, 2015.

[18] Y. Wang and S. Xu, "Gravitational orbit-rotation coupling of a rigid satellite around a spheroid planet," Journal of Aerospace Engineering, vol. 27, no. 1, pp. 140-150, 2014.

[19] Y. Wang and S. Xu, "Relative equilibria of full dynamics of a rigid body with gravitational orbit-attitude coupling in a uniformly rotating second degree and order gravity field," Astrophysics and Space Science, vol. 354, no. 2, pp. 339-353, 2014.

[20] Y. Wang, S. Xu, and L. Tang, "On the existence of the relative equilibria of a rigid body in the J2 problem," Astrophysics and Space Science, vol. 353, no. 2, pp. 425-440, 2014.

[21] Y. Wang, S. Xu, and M. Zhu, "Stability of relative equilibria of the full spacecraft dynamics around an asteroid with orbit-attitude coupling," Advances in Space Research, vol. 53, no. 7, pp. 10921107, 2014.

[22] C. Früh, T. M. Kelecy, and M. K. Jah, "Coupled orbit-attitude dynamics of high area-to-mass ratio (HAMR) objects: influence of solar radiation pressure, earth's shadow and the visibility in light curves," Celestial Mechanics and Dynamical Astronomy, vol. 117, no. 4, pp. 385-404, 2013.

[23] C. Früh and M. K. Jah, "Coupled orbit-attitude motion of high area-to-mass ratio (HAMR) objects including efficient selfshadowing," Acta Astronautica, vol. 95, no. 1, pp. 227-241, 2014.

[24] J. Zhang, S. Zhao, and Y. Yang, "Characteristic analysis for elliptical orbit hovering based on relative dynamics," IEEE Transactions on Aerospace and Electronic Systems, vol. 49, no. 4, pp. 2742-2750, 2013.

[25] J. Zhang, S. Zhao, and Y. Zhang, "Autonomous guidance for rendezvous phasing based on special-point-based maneuvers," Journal of Guidance, Control, and Dynamics, vol. 38, no. 4, pp. 578-586, 2015. 
[26] H. Gui and G. Vukovich, "Finite-time output-feedback position and attitude tracking of a rigid body," Automatica, vol. 74, pp. 270-278, 2016.

[27] A. Sanyal, N. Nordkvist, and M. Chyba, "An almost global tracking control scheme for maneuverable autonomous vehicles and its discretization," IEEE Transactions on Automatic Control, vol. 56, no. 2, pp. 457-462, 2011.

[28] F. Bullo and R. M. Murray, "Proportional derivative (PD) control on the Euclidean group," in Proceedings of the European Control Conference, vol. 2, pp. 1091-1097, European Control Association, Zurich, Switzerland, 1995.

[29] Y. Wang and S. Xu, "Body-fixed orbit-attitude hovering control over an asteroid using non-canonical Hamiltonian structure," Acta Astronautica, vol. 117, pp. 450-468, 2015.

[30] Y. Wang and S. Xu, "Orbital dynamics and equilibrium points around an asteroid with gravitational orbit-attitude coupling perturbation," Celestial Mechanics \& Dynamical Astronomy, vol. 125, no. 3, pp. 265-285, 2016. 


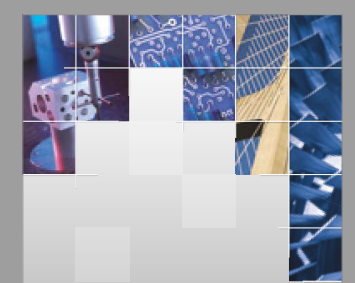

\section{Enfincering}
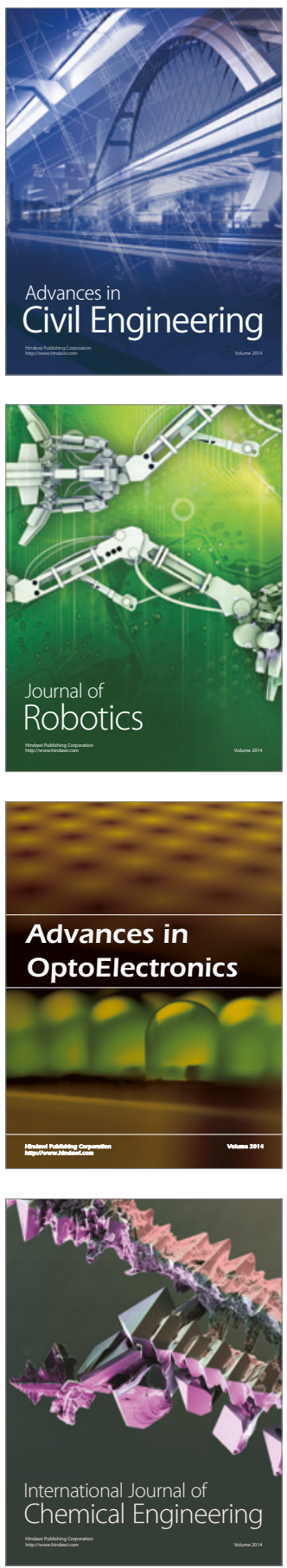

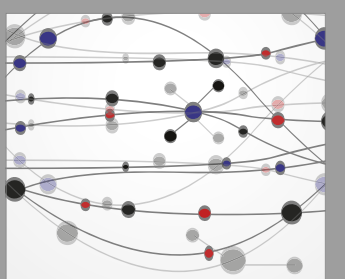

The Scientific World Journal

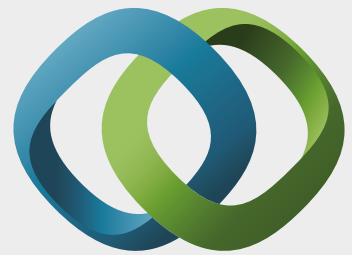

\section{Hindawi}

Submit your manuscripts at

https://www.hindawi.com
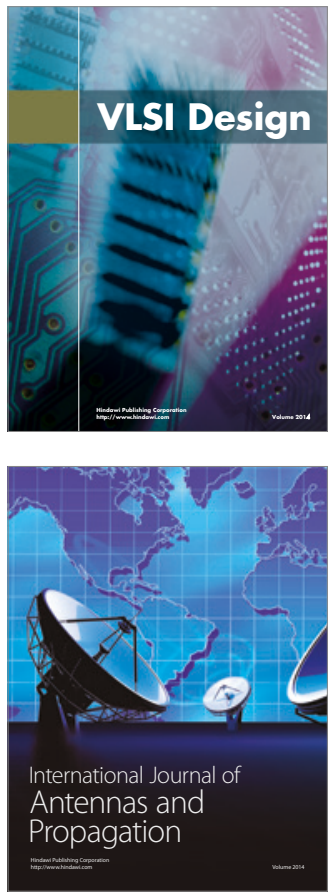

\section{Rotating}

Machinery
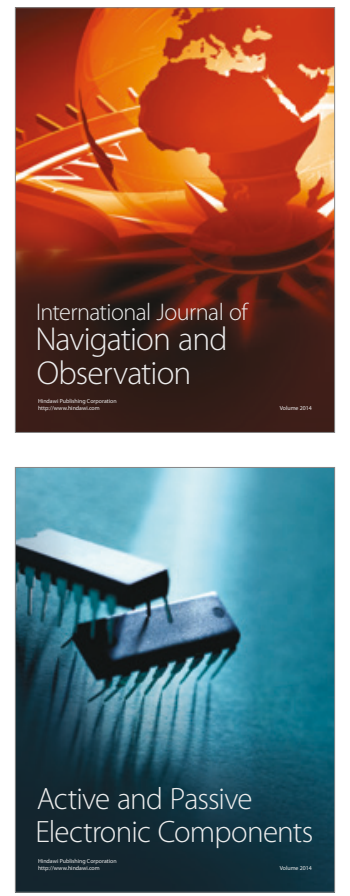
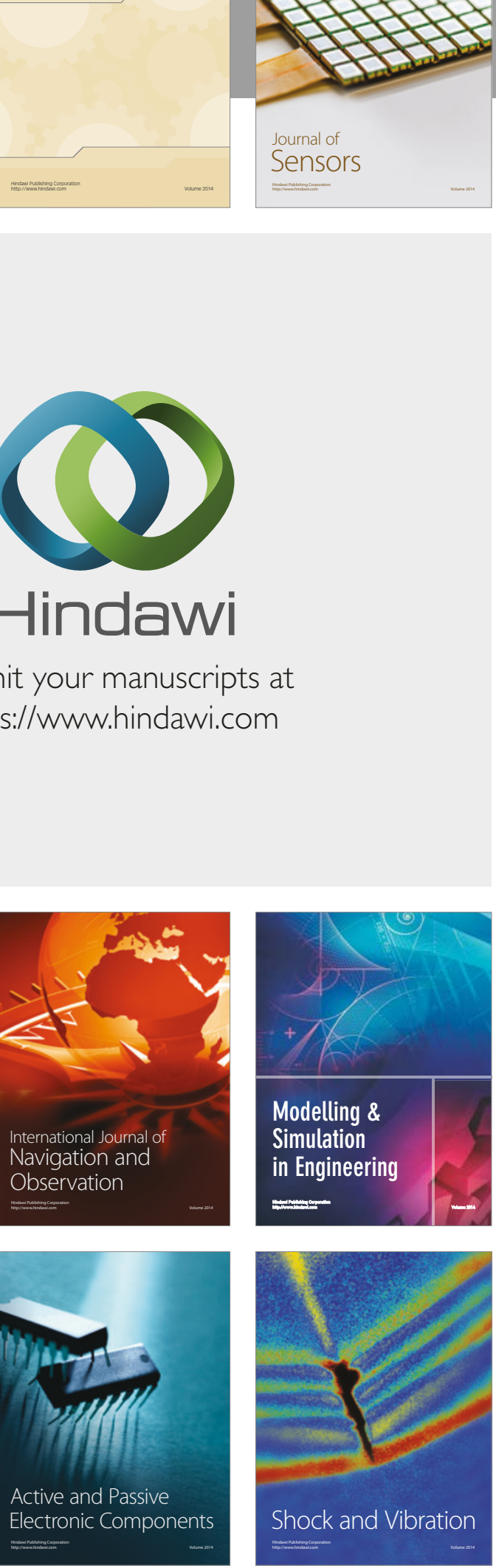
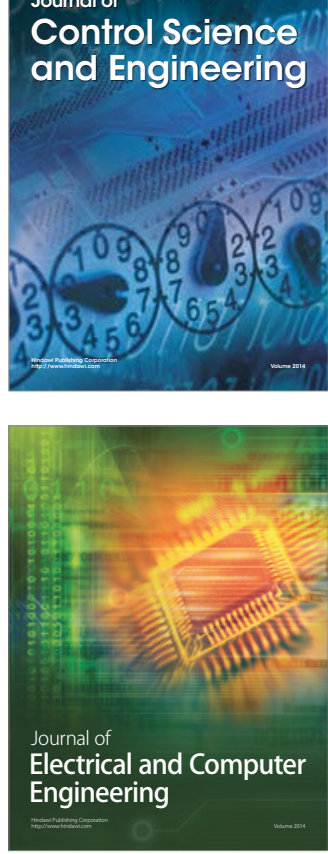

Distributed

Journal of

Control Science

and Engineering
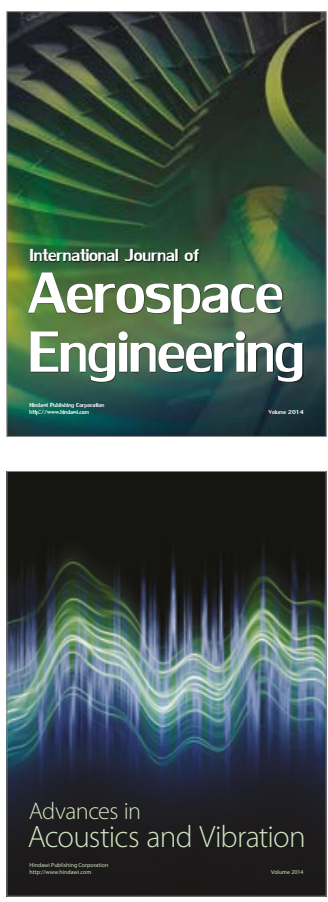

Sensor Networks 